\title{
Algorithms for the computation of moduli spaces for semiquasihomogeneous singularities
}

\author{
Thomas BAYER \\ Institut für Informatik \\ Technische Universität München \\ 80290 München, Germany \\ bayert@in.tum.de \\ www14.in.tum.de/personen/bayert
}

Recibido: 21 de Enero de 2001

Aceptado: 8 de Mayo de 2002

\begin{abstract}
We present algorithms and their implementation in the computer algebra system Singular 2.0 for the computation of equations for moduli spaces for semiquasihomogeneous singularities w.r.t. right equivalence. In addition, we describe the structure of the stabilizer group of Brieskorn-Pham singularities.
\end{abstract}

2000 Mathematics Subject Classification: 14J17, $13 \mathrm{P} 10$.

Key words: Semiquasihomogeneous singularities, Brieskorn-Pham singularities.

\section{Introduction}

One of the important achievements of singularity theory was the classification of some "generic" classes of hypersurface singularities via normal forms by V.I. Arnold, cf. Ch.15 in [2]. For more complicated classes of singularities the classification by normal forms seems to be impossible. In 1997 Greuel, Hertling, and Pfister came up with a geometric classification of semiquasihomogeneous hypersurface singularities with fixed part w.r.t. right- and contact equivalence by geometric methods, i.e., by providing a construction of moduli spaces for such singularities with some invariants fixed, cf. [9]. The moduli spaces w.r.t. right equivalence (and fixed principal part) turn out to be quotients of affine varieties by finite groups, which are again algebraic varieties. We describe algorithms and their implementation in the computer-algebra system Singular 2.0 (cf. [10]) for computing equations of these moduli spaces given the principal part. In addition, we provide a structure theorem for the isometry groups of 
Brieskorn-Pham polynomials and its application to the computation of moduli spaces (cf. Ch. 4 in [3]).

The objects to classify are singularities defined by semiquasihomogeneous power series with principal part $f_{0}$ w.r.t. right equivalence, where $f_{0}$ is a quasihomogeneous polynomial which has an isolated singularity at 0 . Right equivalence leads to an algebraic group action of the isometry group of the polynomial $f_{0}$ (leaving the polynomial fixed) on the vector space spanned by the upper monomials of the Milnor algebra of $f_{0}$. The quotient $\mathcal{M}_{f_{0}}$ of the linearization of this action, which is an affine variety, is the desired coarse moduli space for semiquasihomogeneous singularities with principal part $f_{0}$ w.r.t. right equivalence.

We present the algorithms ArnoldAction, LinearizeAction and ModEqn which are the essential parts of the Singular 2.0 libraries rinvar . lib and qhmoduli. lib. Together with the zeroset.lib they provide constructive means to compute equations for $\mathcal{M}_{f_{0}}$ given the principal part $f_{0}$. The design and implementation of these three libraries is part of the author's diploma thesis, cf. [3].

\section{Acknowledgments}

The present work is based on the author's diploma thesis written at the Fachbereich Mathematik, Universität Kaiserslautern. The author is very grateful to Christoph Lossen and Josef Schicho for useful discussions, to Hans Schönemann for the SinGULAR support, to Prof. Gert-Martin Greuel, the thesis advisor, for numerous discussions and his generous support, and to the anonymous referee for the useful comments, particularly concerning Section 3.2.

\section{Basic notation}

In this section we provide necessary definitions from singularity theory and introduce unfoldings of power series. We do not introduce moduli spaces ${ }^{1}$ since the existence of a moduli space is proved in [9] and technical details of the proof are not important for the computation of the equations.

In the sequel we denote the ring of convergent power series over $\mathbf{C}$ by $\mathbf{C}\left\{x_{1}, x_{2}, \ldots, x_{n}\right\}$. Let $0 \neq f \in \mathbf{C}\left\{x_{1}, x_{2}, \ldots, x_{n}\right\}$ be s.t. $f(0)=0$. The power series $f$ has a singularity at 0 if $\frac{\partial f}{\partial x_{1}}(0)=\frac{\partial f}{\partial x_{2}}(0)=\ldots=\frac{\partial f}{\partial x_{n}}(0)=0$. The Jacobi ideal of $f$ is $j(f):=\left\langle\frac{\partial f}{\partial x_{i}}: 1 \leq i \leq n\right\rangle$ and the Milnor algebra of $f$ is defined by $M_{f}:=\mathbf{C}\left\{x_{1}, x_{2}, \ldots, x_{n}\right\} / j(f)$. The Milnor number $\mu_{f}$ of $f$ is $\mu_{f}:=\operatorname{dim}_{\mathbf{C}} M_{f}$. If there exists a neighborhood $U \subset \mathbf{C}^{n}$ of 0 s.t. 0 is the only singular point of $f$ in $U$ then 0 is called an isolated singularity of $f$. Note that the Milnor number gives an important characterization of isolated singularities, namely $\mu_{f}<\infty$ iff 0 is an isolated singularity of $f$.

\footnotetext{
${ }^{1}$ We refer to Ch. 1 of [12] for a description or to, e.g., [9] for a definition.
} 
In the sequel we fix a weight vector $\mathbf{w}=\left(w_{1}, w_{2}, \ldots, w_{n}\right) \in \mathbf{N}_{+}^{n}$. A monomial $\mathbf{x}^{\alpha}:=\prod_{i=1}^{n} x_{i}^{\alpha_{i}}$ is quasihomogeneous of type $(d ; \mathbf{w})$ if $\operatorname{deg}_{\mathbf{w}}\left(\mathbf{x}^{\alpha}\right):=\left|\mathbf{x}^{\alpha}\right|_{\mathbf{w}}:=$ $\sum_{i=1}^{n} w_{i} \alpha_{i}$. A polynomial $f$ is quasihomogeneous of type $(d ; \mathbf{w})$ if all of its monomials are quasihomogeneous of type $(d ; \mathbf{w})$. For a power series $0 \neq f \in \mathbf{C}\left\{x_{1}, x_{2}, \ldots, x_{n}\right\}$ we define $\operatorname{ord}_{\mathbf{w}}(f)=\min \left\{\left|\mathbf{x}^{\alpha}\right|_{\mathbf{w}}: \mathbf{x}^{\alpha}\right\}$ and $\operatorname{ord}_{\mathbf{w}}(0):=-1$. The power series $f$ is called semiquasihomogeneous of type $(d ; \mathbf{w})$ with principal part $f_{0}$ if $f_{0}$ is a quasihomogeneous polynomial of type $(d ; \mathbf{w})$ with an isolated singularity at 0 and $f=f_{0}$ or $\operatorname{ord}_{\mathbf{w}}\left(f-f_{0}\right)>d$.

By $\operatorname{Aut}\left(\mathbf{C}\left\{x_{1}, x_{2}, \ldots, x_{n}\right\}\right)$ we denote the group of local $\mathbf{C}$-algebra automorphisms. Two power series $f, g \in \mathbf{C}\left\{x_{1}, x_{2}, \ldots, x_{n}\right\}$ are right equivalent, denoted by $f \sim_{r} g$, if there exists a $\varphi \in \operatorname{Aut}\left(\mathbf{C}\left\{x_{1}, x_{2}, \ldots, x_{n}\right)\right.$ s.t. $f=\varphi(g)$. The power series $f \in \mathbf{C}\left\{x_{1}, x_{2}, \ldots, x_{n}\right\}$ is $k$-determined w.r.t. $\sim_{r}$ if for $g \in \mathbf{C}\left\{x_{1}, x_{2}, \ldots, x_{n}\right\}$ we have $f^{(k)}=g^{(k)} \leftrightarrow f \sim_{r} g$ where $f^{(k)}, g^{(k)}$ denote the $k$-jet $(f, g$ truncated at degree $k$ ) respectively.

Theorem 1. Let $f \in \mathbf{C}\left\{x_{1}, x_{2}, \ldots, x_{n}\right\}$ be a power series with an isolated singularity at 0 . Then $f$ is $\left(\mu_{f}+1\right)$-determined.

Proof. We refer to Section 6.3 of [2] (p. 121ff).

For the construction of a moduli space we need the notion of families of unfoldings of negative weight. Let $f \in \mathbf{C}\left\{x_{1}, x_{2}, \ldots, x_{n}\right\}$ be semiquasihomogeneous with principal part $f_{0}$. The power series $f$ can be considered as a function germ $f:\left(\mathbf{C}^{n}, 0\right) \longrightarrow(\mathbf{C}, 0)$ which can be deformed as follows. An unfolding of $f$ over a germ $(T, t)$ is a map $F:\left(\mathbf{C}^{n}, 0\right) \times(T, t) \longrightarrow(\mathbf{C}, 0)$ s.t. the following diagram commutes,

$$
\begin{array}{cccc}
\left(\mathbf{C}^{n}, 0\right) & \hookrightarrow & \left(\mathbf{C}^{n}, 0\right) \times(T, t) \\
\downarrow f & & \downarrow \phi \\
(\mathbf{C}, 0) & \hookrightarrow & (\mathbf{C}, 0) \times(T, t) \\
\downarrow & & \downarrow \\
0 & \hookrightarrow & (T, t)
\end{array}
$$

where $\phi(x, t)=(F(x, t), t)$, and $F(x, t)=f(x)+g(x, t)$ for some $g \in \mathbf{C}^{n}\{\mathbf{x}, \mathbf{t}\}$ with $g(x, 0)=0$. Two unfoldings $F, F^{\prime}$ of $f$ are right equivalent if there exists an isomorphism $\Psi:\left(\mathbf{C}^{n}, 0\right) \times(T, 0) \rightarrow\left(\mathbf{C}^{n}, 0\right) \times(T, 0), \Psi(x, t)=(\psi(x, t), t)$ s.t. $F(\psi(x, t), t)=F^{\prime}(x, t)$. A morphism $\varphi:(S, s) \rightarrow(T, t)$ induces an unfolding $\varphi^{*} F:\left(\mathbf{C}^{n}, 0\right) \times(S, s) \rightarrow(\mathbf{C}, 0)$ of $f$ by $\varphi^{*} F\left(x, s^{\prime}\right)=F\left(x, \varphi\left(s^{\prime}\right)\right)$ for $s^{\prime} \in(S, s)$ (base change). The unfolding $F$ is semiuniversal if for each unfolding $G$ of $f$ over some base space there exist a base space $(S, s)$ and a morphism $\varphi:(S, s) \rightarrow(T, t)$ s.t. the unfolding $G$ is right equivalent to the induced unfolding $\varphi^{*} F$ and the differential $d \varphi$ is unique. $F$ is called an unfolding of negative weight over $(T, 0)$ if $F \sim_{r} f^{\prime}(x)+g(x, t)$ for some semiquasihomogeneous power series $f^{\prime}$ with $g(x, 0)=0$ and $\operatorname{deg}_{x}(g)>d$. If the germ $(T, t)$ is replaced by a base space $T$ then the map $F:\left(\mathbf{C}^{n}, 0\right) \times T \longrightarrow(\mathbf{C}, 0)$ is called a family of unfoldings of negative weight over $S$. Hence $\phi(x, t)=(F(x, t), t)=\left(F_{t}(x), t\right)$ and for each $t \in T$ the germ 
$F:\left(\mathbf{C}^{n}, 0\right) \times(T, t) \longrightarrow(\mathbf{C}, 0)$ is an unfolding of negative weight of $F_{t}$ over $(T, t)$. If, in addition, for any $t \in T, F_{t}:\left(\mathbf{C}^{n}, 0\right) \times(T, t) \longrightarrow(\mathbf{C}, 0)$ is right equivalent to a semiquasihomogeneous power series with principal part $f_{0}$ then $F$ is called a family of unfoldings of negative weight with principal part $f_{0}$ or a family of $f_{0}^{-}$-unfoldings for short. For families of unfoldings right equivalence, base change and semiuniversality are defined as for unfoldings.

For $B$ a monomial basis of the Milnor algebra of $f_{0}\left(|B|<\infty\right.$ since $\left.\mu_{f_{0}}<\infty\right)$ we define the set of upper monomials $B_{-}=\left\{m_{1}, m_{2}, \ldots, m_{k}\right\}=\left\{m \in B: \operatorname{deg}_{\mathbf{w}}(m)>\right.$ $d\}$ of $M_{f_{0}}$ and the space $T_{-}=\mathbf{C}^{k}$. Given $f_{0}$, we define the family of $f_{0}^{-}-$unfoldings

$$
\begin{aligned}
F & : \quad \mathbf{C}^{n} \times T_{-} \longrightarrow \mathbf{C}^{n}, \\
F(x, t) & =f_{0}(x)+\sum_{i=1}^{k} t_{i} m_{i}
\end{aligned}
$$

and call it the semiuniversal family of unfoldings of negative weight of semiquasihomogeneous power series with principal part $f_{0}$. This name is justified by Proposition 3.4.5 and Theorem 3.2.3 below.

Example 1. The polynomial $f_{0}=x^{2} y+x^{2} z+y^{5}-z^{5}$ is semiquasihomogeneous of type $(5 ; 2,1,1)$. The Milnor number equals $\mu_{f_{0}}=24$, hence $f_{0}$ has an isolated singularity at 0 . Upper monomials are given by $B_{-}=\left\{y^{3} z^{3}, x^{2} y^{3}, x^{2} y^{2}\right\}$ and the family of $f_{0}^{-}$-unfoldings is

$$
F\left(x, y, z, t_{1}, t_{2}, t_{3}\right)=x^{2} y+x^{2} z+y^{5}-z^{5}+t_{1} y^{3} z^{3}+t_{2} x^{2} y^{2}+t_{3} x^{2} y^{3}
$$

\section{Existence of a coarse moduli space}

We briefly describe the construction of a coarse moduli space for semiquasihomogeneous power series with principal part $f_{0}$ given by Greuel, Hertling and Pfister in [9]. The building blocks are a Theorem of Greuel, Hertling and Pfister on the order of quasihomogeneous maps and a Theorem of Arnold on semiquasihomogeneous power series, which states that each semiquasihomogeneous power series with principal part $f_{0}$ is right equivalent to some $F_{t}$ for $t \in T_{-}$. By [9] we only need to consider isomorphisms of order $\geq 0$, hence we can construct, by comparing coefficients, the isometry group of $f_{0}$ and its induced action on $T_{-}$, the base space of the semiuniversal unfolding $F$, which turns out to be a finite group action. [9] proved that the quotient of this action is a coarse moduli space for semiquasihomogeneous power series with principal part $f_{0}$ w.r.t. right equivalence.

We only provide those proofs, which are important for the algorithms of Section 4 or which are not given in [9]. 


\subsection{The order of maps}

Let $R=\mathbf{C}\left\{x_{1}, x_{2}, \ldots, x_{n}\right\}$ be the ring of convergent power series, let $A u t(R)$ be the group of local $\mathbf{C}$-algebra automorphisms of $R$ and let $\mathbf{w}=\left(w_{1}, w_{2}, \ldots, w_{n}\right) \in \mathbf{N}^{n}$ be weights. By $R_{d}$ we denote the ideal generated by all power series of weighted order greater or equal to $d$.

Definition 1. (a) Let $i d \neq \varphi: R \rightarrow R$ be a ring homomorphism. The weighted order of $\varphi$, denoted by $\operatorname{ord}_{\mathbf{w}}(\varphi)$, is the maximal integerd s.t. $\operatorname{ord}_{\mathbf{w}}\left(\varphi\left(x_{i}\right)-x_{i}\right) \geq$ $w_{i}+d$ for $1 \leq i \leq n$.

(b) For $d \geq 0$ we define Aut $t_{\geq d}(R):=\left\{\varphi \in \operatorname{Aut}(R): \operatorname{ord}_{\mathbf{w}}(\varphi) \geq d\right\} \cup\{i d\}$ and $\operatorname{Aut}_{>d}(R):=A u t_{\geq d+1}(R)$.

If $f$ is semiquasihomogeneous of type $(d ; \mathbf{w})$ and $\varphi$ is an automorphism of $\mathbf{C}\left\{x_{1}, x_{2}, \ldots, x_{n}\right\}$ of order 0 then $\varphi(f)$ is semiquasihomogeneous of type $(d ; \mathbf{w})$. The converse, due to Greuel, Hertling and Pfister (cf. Theorem 2.1 in [9]), is by no means trivial and plays an important role for the construction of the moduli space.

Theorem 2. [9] Let $f$ and $g$ be semiquasihomogeneous power series of type $(d ; \mathbf{w})$ and let $\varphi \in \operatorname{Aut}\left(\mathbf{C}\left\{x_{1}, x_{2}, \ldots, x_{n}\right\}\right)$. Then

$$
f=\varphi(g) \Longrightarrow \operatorname{ord}_{\mathbf{w}}(\varphi) \geq 0
$$

Proof. We refer to [9], Theorem 2.1.

Hence it suffices to consider only automorphisms $\varphi \in \operatorname{Aut}(R)$ which satisfy $\operatorname{ord}_{\mathbf{w}}(\varphi) \geq 0$. For the subgroups $A u t_{\geq k}$ we have the following result.

Proposition 1. For $q>p \geq 0 A u t_{\geq q}(R) \subset A u t_{\geq p}(R)$ is a normal subgroup.

Proof. We refer to Proposition 12.4.2 in [2] (p. 203).

Note that if $f$ is semiquasihomogeneous with principal part $f_{0}$ then right equivalence need not preserve the principal part.

\subsection{Arnold's Theorem on semiquasihomogeneous power series}

In this section we present a result of V.I. Arnold, cf. [1] (or Ch. 12.6, in [2], p.209), which says that any semiquasihomogeneous power series with principal part $f_{0}$ is right equivalent to $f_{0}+\sum_{\alpha \in B_{-}} c_{\alpha} \mathbf{x}^{\alpha}$ for some complex numbers $c_{\alpha}$. Unfortunately, they are not unique in general.

The proof of Arnold's Theorem provides a construction of a morphism of weighted order $>0$ which maps a given semiquasihomogeneous power series (with terms of arbitrarily high degree) with principal part $f_{0}$ to some $F(\mathbf{x}, \mathbf{c}), \mathbf{c} \in T_{-}$, where $F$ is the semiuniversal family of $f_{0}^{-}-$unfoldings. This construction is of fundamental 
importance for the existence of an induced action of the stabilizer $G_{f_{0}}^{\mathbf{w}}$ of $f_{0}$ on $T_{-}$. We follow the presentation in Ch. 12.5 and 12.6 of [2] (p. 206ff).

In the sequel let $f_{0} \in \mathbf{C}\left[x_{1}, x_{2}, \ldots, x_{n}\right]$ be a quasihomogeneous polynomial of type $(d ; \mathbf{w})$ with an isolated singularity at 0 . The following Lemma will be needed to prove Arnold's Theorem.

Lemma 1. (a) Let $f$ be a power series of weighted order $d$ and $\varphi$ be an automorphism of the form $\varphi(\mathbf{x})=\mathbf{x}-\mathbf{v}$ of order $\delta>0, \mathbf{v}=\left(v_{1}, v_{2}, \ldots, v_{n}\right)$. Then

$$
\varphi(f)=f-\sum \frac{\partial f}{\partial x_{i}} v_{i}+r
$$

for some $r$ s.t. $\operatorname{ord}_{\mathbf{w}}(r)>d+\delta$.

(b) Any semiquasihomogeneous power series $f_{0}+f_{1}$ with fixed part $f_{0}$ and $\operatorname{ord}_{\mathbf{w}}\left(f_{1}\right)>$ $d$ and $d^{\prime}>d$ can be transformed by a polynomial diffeomorphism of order $>0$ to $f_{0}+f_{1}^{\prime}$ s.t. the terms of degree less than $d^{\prime}$ of $f_{1}$ and $f_{1}^{\prime}$ coincide, and the terms of $f_{1}^{\prime}$ of degree $d^{\prime}$ reduce to $\sum_{\alpha \in B_{-},|\alpha|_{\mathrm{w}}=d^{\prime}} c_{\alpha} \mathbf{x}^{\alpha}$ for some $c_{\alpha} \in \mathbf{C}$.

Proof. (a) We refer to the Lemma in Ch. 12.5 in [2] (p.207).

(b) Let $g_{1}$ denote the sum of all terms of degree $d^{\prime}$ in $f_{1}$, let $g_{1}^{\prime}$ denote the terms of $f_{1}$ of degree $<d^{\prime}$ and set $g_{1}^{\prime \prime}=f_{1}-g_{1}-g_{1}^{\prime}$. Since $\left\{\mathbf{x}^{\alpha}: \alpha \in B_{-},|\alpha|_{\mathbf{w}}=d^{\prime}\right\}$ is a basis of monomials of $M_{f_{0}}$ of degree $d^{\prime}$ we can write $g_{1}$ as

$$
g_{1}=\sum_{i=1}^{n} \frac{\partial f_{0}}{\partial x_{i}} v_{i}+\sum_{\alpha \in B-,|\alpha|_{\mathbf{w}}=d^{\prime}} c_{\alpha} \mathbf{x}^{\alpha}
$$

for some $c_{\alpha} \in \mathbf{C}$. Since $g_{1}$ is quasihomogeneous, $v_{1}, v_{2}, \ldots, v_{n}$ can be chosen to be quasihomogeneous of degree $d^{\prime}-d+w_{1}, d^{\prime}-d+w_{2}, \ldots, d^{\prime}-d+w_{n}$ respectively. Consider the morphism $\varphi$ defined by $\varphi\left(x_{i}\right)=x_{i}-v_{i}$. We have $\operatorname{ord}_{\mathbf{w}}\left(\varphi\left(x_{i}\right)-x_{i}\right)=$ $\operatorname{ord}_{\mathbf{w}}\left(v_{i}\right)=w_{i}+d^{\prime}-d$, so the order of $\varphi$ equals $d^{\prime}-d>0$. By applying (a) to $f=f_{0}+f_{1}$ we obtain

$$
\begin{aligned}
\varphi(f)=f_{0} & -\sum_{i=1}^{n} \frac{\partial f_{0}}{\partial x_{i}} v_{i}+r_{0}+f_{1}-\sum_{i=1}^{n} \frac{\partial f_{1}}{\partial x_{i}} v_{i}^{\prime}+r_{1} \\
=f_{0} & +\sum_{\alpha \in B_{-},|\alpha|_{\mathbf{w}}=d^{\prime}} c_{\alpha} \mathbf{x}^{\alpha}+g_{1}^{\prime} \\
& +\left(r_{0}+g_{1}^{\prime \prime}-\sum_{i=1}^{n} \frac{\partial f_{1}}{\partial x_{i}} v_{i}+r_{1}\right)
\end{aligned}
$$

where the (weighted) order of the terms in the bracket is greater than $d^{\prime}$.

Theorem 3. [1] Let $f$ be a semiquasihomogeneous power series with principal part $f_{0}$. Then

$$
f \sim_{r} f_{0}+\sum_{\alpha \in B_{-}} c_{\alpha} \cdot \mathbf{x}^{\alpha} .
$$


for some $c_{\alpha} \in \mathbf{C}$.

Proof. Let $d^{\prime}>d$ be the minimal degree of a term in $f$ not appearing in $f_{0}$ and set $d^{\prime \prime}:=\max \left\{|\alpha|_{\mathbf{w}}: \alpha \in B_{-}\right\}$. We use Lemma 3.2.1(a) to obtain a diffeomorphism $\varphi_{1}$ s.t. $\operatorname{ord}_{\mathbf{w}}\left(\varphi_{1}\right) \geq d^{\prime}-d$ and $\varphi_{1}(f)=f_{0}+\sum_{\alpha \in B_{-},|\alpha|_{\mathbf{w}}<d^{\prime}+1} c_{\alpha} \mathbf{x}^{\alpha}+f_{1}$ where $\operatorname{ord}_{w}\left(f_{1}\right)>d^{\prime}$. We successively apply Lemma 3.2.1(b) and obtain in the $k$-th step

$$
\varphi_{k} \circ \varphi_{k-1} \circ \ldots \circ \varphi_{1}(f)=f_{0}+\sum_{\alpha \in B_{-},|\alpha|_{\mathbf{w}}<d^{\prime}+k} c_{\alpha} \mathbf{x}^{\alpha}+f_{k}
$$

where $\operatorname{ord}_{\mathbf{w}}\left(f_{k}\right) \geq d^{\prime}+k$. Note that $\varphi_{k}$ does not change the coefficients $c_{\alpha}$ where $|\alpha|_{\mathbf{w}}<d^{\prime}+k$. If $d^{\prime}+k>d^{\prime \prime}$ then no new terms of the form $c_{\alpha} \mathbf{x}^{\alpha}, \alpha \in B_{-}$, are introduced. Since $f$ has an isolated singularity at the origin we have $\mu(f)<\infty$ by Theorem 2.1. Note that the Milnor numbers of $f$ and $f_{0}$ coincide, hence there exists $N>0$ s.t. $\operatorname{ord}\left(f_{N}\right)>\mu\left(f_{0}\right)$ (not the weighted order) and therefore

$$
f_{0}+\sum_{\alpha \in B-,|\alpha|_{w}<d^{\prime}+k} c_{\alpha} \mathbf{x}^{\alpha} \sim_{r} \varphi_{N} \circ \varphi_{N-1} \circ \ldots \circ \varphi_{1}(f) \sim_{r} f
$$

via some map $\varphi \in \operatorname{Aut}(\mathbf{C}\{\mathbf{x}\})$.

In the next section, Arnold's Theorem will be used to construct a group action on $T_{-}$, where the order of the morphism, constructed in the proof above, is of fundamental importance.

Remark 1. (a) Arnold's Theorem provides a correspondence between semiquasihomogeneous power series with principal part $f_{0}$ and points of $T_{-}$. Unfortunately, this correspondence is not unique which can be seen, e.g., by applying the $\mathbf{C}^{*}$-action (which is of order 0 ) of $f_{0}$ to $F$. For maps of order $>0$ the correspondence is unique, as shown in Proposition 2.3 in [9].

(b) By Proposition 2.3 of [9], the map $\varphi$ constructed in the proof of Arnold's Theorem has order $>0$.

(c) In the proof of Arnold's Theorem it suffices to compute the morphisms $\varphi_{k}$ for $1 \leq k \leq d^{\prime \prime}-d^{\prime}+1$ because we are only interested in the coefficients $c_{\alpha}$ and

$$
\varphi_{d^{\prime \prime}-d^{\prime}+1} \circ \ldots \circ \varphi_{1}(f)=f_{0}+\sum_{\alpha \in B_{-},|\alpha|_{\mathbf{w}}} c_{\alpha} \mathbf{x}^{\alpha}+f_{d^{\prime \prime}-d^{\prime}+1}
$$

and $\operatorname{ord}_{\mathbf{w}}\left(f_{d^{\prime \prime}-d^{\prime}+1}\right)>d^{\prime \prime}$. The application of $\varphi_{k}$ for $k>d^{\prime \prime}-d^{\prime}+1$ increases the weighted order of $f_{k^{\prime}-1}$ and does not change $f_{0}+\sum_{\alpha \in B_{-},|\alpha|_{\mathrm{w}}} c_{\alpha} \mathbf{x}^{\alpha}$.

Example 2. For $f_{0}=x^{2} z+x^{2} y-z^{5}+y^{5}$ we have to apply Arnold's Theorem to $f=f_{0}+x^{2} z^{3}$ because $x^{2} z^{3}$ is no upper monomial, i.e., $f(\mathbf{x}) \neq F\left(\mathbf{x}, t_{0}\right)$ for all $t_{0}$. From $x^{2} z^{3}=\frac{1}{2}\left(x z^{2}-x y z+x y^{2}\right)\left(\frac{\partial f_{0}}{\partial x}\right)-x^{2} y^{3}$ we obtain the map $\varphi(x)=$ 
$x-\frac{1}{2}\left(x z^{2}-x y z+x y^{2}\right), \varphi(y)=y, \varphi(z)=z$. Application yields

$$
\begin{aligned}
\varphi(f)=\quad & f_{0}-x^{2} y^{3} \\
& -\frac{3}{4} x^{2} z^{5}+\frac{3}{4} x^{2} y z^{4}-\frac{3}{4} x^{2} y^{2} z^{3}+\frac{1}{4} x^{2} y^{3} z^{2}-\frac{1}{4} x^{2} y^{4} z+\frac{1}{4} x^{2} y^{5} \\
& \frac{1}{4} x^{2} z^{7}-\frac{1}{2} x^{2} y z^{6}+\frac{3}{4} x^{2} y^{2} z^{5}-\frac{1}{2} x^{2} y^{3} z^{4}+\frac{1}{4} x^{2} y^{4} z^{3}
\end{aligned}
$$

Since the terms of the last two lines have degree $>7=\max \left\{\operatorname{deg}_{\mathbf{w}}(m): m \in B_{-}\right\}$we obtain $f \sim_{r} f_{0}-x^{2} y^{3}$ by Theorem 3.2.3 and Remark 3.2.1(c).

\subsection{The induced group action}

Any morphism $\sigma \in \operatorname{Aut}(\mathbf{C}\{\mathbf{x}\})$ which leaves $f_{0}$ invariant induces a map $\Theta(\sigma): T_{-} \rightarrow$ $T_{-}$by Arnold's Theorem. [9] proved that $\Theta$ induces an algebraic group action on $T_{-}$when applied to $F$ and that morphisms of order $>0$ preserve uniqueness (cf. the proposition below). For the construction of the moduli space we may therefore restrict ourselves to the factor group $A u t_{>0}(\mathbf{C}\{\mathbf{x}\}) / A u t_{>0}(\mathbf{C}\{\mathbf{x}\})$ which is isomorphic to the group of quasihomogeneous morphisms of $\mathbf{C}\{\mathbf{x}\}$. Additionally, [9] showed that the group action induced by $\Theta$ on $T_{-}$is finite.

Proposition 2. [9] For any semiquasihomogeneous power series $f$ with principal part $f_{0}$ there exists an automorphism $\psi \in A_{u} t_{>0}(\mathbf{C}\{\mathbf{x}\})$ and a $t_{0} \in T_{-}$s.t. $\psi(f)=F_{t_{0}}$. If $\psi^{\prime} \in$ Aut $_{>0}(\mathbf{C}\{\mathbf{x}\})$ and $\psi^{\prime}\left(F_{t_{0}}\right)=F_{t_{0}^{\prime}}$ for some $t_{0}^{\prime} \in T_{-}$then $t_{0}=t_{0}^{\prime}$.

Proof. We refer to Proposition 2.3 in [9].

Hence only a morphism of order 0 may send a semiquasihomogeneous power series with principal part $f_{0}$ to a different point of $T_{-}$(e.g., the $\mathbf{C}^{*}-$ action of $f_{0}$ ). Note that morphisms of Arnold's Theorem have order $>0$. Therefore we are interested in the following group.

Definition 2. A morphism $\sigma \in \operatorname{Aut}(\mathbf{C}\{\mathbf{x}\})$ is called quasihomogeneous (w.r.t. w) iff $\sigma$ maps quasihomogeneous elements to quasihomogeneous elements of the same degree. Set $G^{\mathbf{w}}:=\{\sigma \in \operatorname{Aut}(\mathbf{C}\{\mathbf{x}\}): \sigma$ is quasihomogeneous w.r.t.w $\}$.

If $\sigma$ is quasihomogeneous then $\sigma\left(x_{i}\right)$ is quasihomogeneous of degree $w_{i}$, i.e., the components $\sigma_{i}$ are quasihomogeneous polynomials. By considering the natural inclusion $i: G^{\mathbf{w}} \hookrightarrow A u t_{\geq 0}(\mathbf{C}\{\mathbf{x}\})$ and the projection $\pi: A_{\geq 0}(\mathbf{C}\{\mathbf{x}\}) \rightarrow$ $A_{u t_{\geq 0}}(\mathbf{C}\{\mathbf{x}\}) / A_{u t}(\mathbf{C}\{\mathbf{x}\})$ one obtains the following result.

Proposition 3. The set $G^{\mathbf{w}}$ is an algebraic group, in particular,

$$
A u t_{\geq 0}(\mathbf{C}\{\mathbf{x}\}) / A u t_{>0}(\mathbf{C}\{\mathbf{x}\}) \cong G^{\mathbf{w}} .
$$


Proof. We refer to Proposition 2 in Ch. 12.4 (p. 203) in [2].

Definition 3. The stabilizer group of $f_{0}$ is $G_{f_{0}}^{\mathbf{w}}:=\left\{\sigma \in G^{\mathbf{w}}: \sigma\left(f_{0}\right)=f_{0}\right\}$.

Note that $G_{f_{0}}^{\mathbf{w}}$ is an algebraic group since it is a closed subgroup of $G^{\mathbf{w}}$. Combining Theorem 3.1.2., Theorem 3.2.3 and the results above one can define a group action of $G_{f_{0}}^{\mathbf{w}}$ on $T_{-}$as follows.

Proposition 4. [9] (a) For any $\sigma \in G_{f_{0}}^{\mathbf{w}}$ and $t \in T_{-}$there exists a unique $s=\Theta(\sigma)(t)$ and an automorphism $\varphi \in$ Aut $_{>0}(\mathbf{C}\{\mathbf{x}\})$ s.t. $\varphi \circ \sigma\left(F_{t}\right)=F_{s}$.

(b) The function $\Theta: G_{f_{0}}^{\mathbf{w}} \rightarrow$ Aut $\left(T_{-}\right)$is a group homomorphism.

(c) The components of $\Theta(\sigma)\left(t_{i}\right)$ are quasihomogeneous polynomials of degree $\operatorname{deg}_{\mathbf{w}}\left(m_{i}\right)-$

d. In particular, the group $\Theta\left(G_{f_{0}}^{\mathbf{w}}\right)$ acts algebraically on $T_{-}$and this action commutes with a $\mathbf{C}^{*}$-action on $T_{-}$.

Proof. We refer to Proposition 2.4 of [9]

The induced group action of $G_{f_{0}}^{\mathbf{w}}$ on $T_{-}=\mathbf{C}^{3}$, where $f_{0}=x^{2} z+x^{2} y-z^{5}+y^{5}$, is given in Example 4.1.4. For the construction of the moduli space we are interested in the image of the stabilizer $G_{f_{0}}^{\mathbf{w}}$ under $\Theta$.

Definition 4. The group $E_{f_{0}}:=\Theta\left(G_{f_{0}}^{\mathbf{w}}\right)$ is the subgroup of Aut $\left(T_{-}\right)$which is induced by the action of $G_{f_{0}}^{\mathbf{w}}$ on $T_{-}$provided by Arnold's Theorem.

[9] proved that the quotient $T_{-} / E_{f_{0}}$ is a coarse moduli space for semiquasihomogeneous power series with fixed principal part.

Remark 2. (a) Unfortunately (for computational purposes) the map $\varphi$ of Proposition 3.3.2 cannot be omitted.

(b) It follows from (a) that, in general, $E_{f_{0}}$ does not act linearly on $T_{-}$, even if $G_{f_{0}}^{\mathbf{w}} \subset G L_{n}(\mathbf{C})$. In order to compute the equations of $T_{-} / E_{f_{0}}$ one has to linearize the action of $E_{f_{0}}$ on $T_{-}$which is the main topic in Section 4.4.2.

The construction of the quotient $T_{-} / E_{f_{0}}$ is considerably simplified by the fact that $E_{f_{0}}$ is finite, hence reductive, so Hilbert's Finiteness Theorem implies that the quotient is an algebraic variety.

Theorem 4. [9] Let $f_{0} \in \mathbf{C}\left[x_{1}, x_{2}, \ldots, x_{n}\right]$ be a quasihomogeneous polynomial of type $(d ; \mathbf{w})$ with an isolated singularity at 0 and set $\bar{w}_{i}:=\frac{w_{i}}{d}$.

(a) The group $G_{f_{0}}^{\mathbf{w}}$ is finite if $\bar{w}_{1}<\frac{1}{2}, \ldots, \bar{w}_{n-1}<\frac{1}{2}, \bar{w}_{n} \leq \frac{1}{2}$.

(b) The group $E_{f_{0}}$ is finite.

Proof. We refer to Proposition 2.7 in [9], or for a more detailed treatment, to Theorem 2.3.56 in [3] (p. 36ff).

Remark 3. Under the above hypotheses it is tempting, but wrong, to conclude that $G_{f_{0}}^{\mathbf{w}} \subset G L_{n}(\mathbf{C})$ or that $\oplus_{i=1}^{n} \mathbf{C} \frac{\partial f_{0}}{\partial x_{i}}$ is a $G_{f_{0}}^{\mathbf{w}}$-module. ${ }^{2}$ Consider the quasihomogeneous

\footnotetext{
$[15]$.

${ }^{2}$ For $G \subseteq G_{f_{0}}^{\mathbf{w}} \cap G L_{n}(\mathbf{C})$ Orlik and Wagreich proved that it is a $G$-module, cf. Lemma 5.3 or
} 
polynomial $f_{0}=x^{2}-x y^{2}+y^{4}$ of type $(4 ; 2,1)$ which has an isolated singularity at $0\left(\mu_{f_{0}}(0)=3\right)$. Its stabilizer group $G_{f_{0}}^{\mathbf{w}}$ has order 8 and is generated by the two automorphisms $\sigma, \tau$, where

$$
\begin{aligned}
& \sigma(x)=-x+y^{2}, \\
& \sigma(y)=y,
\end{aligned}
$$

and $\tau(x)=-x, \tau(y)=i y$. It is obvious that $G_{f_{0}}^{\mathbf{w}} \nsubseteq G_{2}(\mathbf{C})$. Moreover, $\mathbf{C} \frac{\partial f_{0}}{\partial x} \oplus \mathbf{C} \frac{\partial f_{0}}{\partial y}$ is not a $G_{f_{0}}^{\mathbf{w}}$-module since $\sigma^{-1} \bullet \frac{\partial f_{0}}{\partial y}=2 y \frac{\partial f_{0}}{\partial x}+\frac{\partial f_{0}}{\partial y}$. Nonetheless, this is true for Brieskorn-Pham polynomials as we will show in Section 5.

\subsection{Existence of the moduli space}

We present the construction of coarse moduli spaces for semiquasihomogeneous singularities with principal part $f_{0}$ w.r.t. right equivalence given by Greuel, Hertling and Pfister (cf. Section 1 in [9]), whereas we do not introduce the notion of a moduli functor or moduli space. We just mention that the points of a moduli space are in $1: 1$ correspondence with the isomorphism classes of semiquasihomogeneous singularities with principal part $f_{0}$.

Recall that the family $F$ of $f_{0}^{-}$-unfoldings has been called the semiuniversal family of unfoldings of negative weight of semiquasihomogeneous power series with principal part $f_{0}$. This name is justified by the proposition below.

Proposition 5. [9] Let $f$ be a semiquasihomogeneous power series with principal part $f_{0}$.

(a) For each $t \in T_{-}$the restriction of $F$ to the germ $\left(T_{-}, t\right)$ is a semiuniversal unfolding of $F_{t}$ of negative weight.

(b) $T_{-}$does not contain trivial subfamilies.

(c) If $f_{0}$ is neither simple nor simple elliptic then there exist $t, t^{\prime} \in T_{-}$arbitrary close to 0 s.t. $F_{t} \sim_{r} F_{t^{\prime}}$ (that is, the unfolding $F$ is not universal in any neighborhood of $0)$.

Proof. We refer to [9], Remark 1.1, Lemma 1.2 and Remark 1.4.

If the singularity of $f_{0}$ is simple or simple elliptic, K. Saito proved that $T_{-}=\{0\}$, i.e., all power series with principal part $f_{0}$ are right equivalent to $f_{0}$ (cf. [17]).

The functor for our moduli problem is defined as follows where $\tau=\operatorname{alg}$ if $\mathcal{S}$ denotes the category of algebraic spaces and $\tau=h o l$ if $\mathcal{S}$ denotes the category of complex spaces.

$$
\begin{aligned}
U n f_{f_{0}, \tau}^{-} & : \mathcal{S} \longrightarrow(\text { sets }), \\
S & \in \operatorname{Obj}(\mathcal{S}) \longmapsto\left\{[\phi]_{\sim_{r}}: \phi \text { a family of } f_{0}^{-}-\text {unfoldings over } S\right\}, \\
T \stackrel{\varphi}{\rightarrow} S & \in \operatorname{Mor}(T, S) \longmapsto\left([\phi]_{\sim_{r}} \mapsto\left[\varphi^{*} \phi\right]_{\sim_{r}}\right) .
\end{aligned}
$$


Proposition 6. For the reduced point $\{p t\} \in \mathcal{S}$ we obtain

$$
U n f_{f_{0}, \tau}^{-}(\{p t\})=\left\{[f]_{\sim_{r}}: f \text { semiquasihomogeneous with principal part } f_{0}\right\} .
$$

Proof. By definition, a family $\phi$ of negative weight with principal part $f_{0}$ over $\{p t\}$ is a quasihomogeneous power series with principal part $f_{0}$.

By Theorem 3.2.3 a quasihomogeneous power series $f$ is right equivalent to $F_{t}$ for some $t \in T_{-}$. Hence $U n f_{f_{0}, \tau}^{-}(\{p t\})$ is the desired set of equivalence classes classifying semiquasihomogeneous hypersurface singularities with principal part $f_{0}$ w.r.t. right equivalence.Greuel, Hertling and Pfister solved the geometric classification problem by providing a coarse moduli space for $U n f_{f_{0}, \tau}^{-}$.

Theorem 5. [9]. Let $f_{0} \in \mathbf{C}\left[x_{1}, x_{2}, \ldots, x_{n}\right]$ be a quasihomogeneous polynomial with an isolated singularity at 0 . The quotient

$$
\mathcal{M}_{f_{0}, \tau}:=T_{-} / E_{f_{0}}
$$

is a coarse moduli space for the functor $U n f_{f_{0}, \tau}^{-}$where $\tau=$ alg/hol.

Proof. We refer to Theorem 1.3 and Remark 1.5 in [9].

Note that the quotient $\mathcal{M}_{f_{0}, \text { alg }}$ is an affine variety since $E_{f_{0}}$ is finite and acts algebraically on $T_{-}$.

Example 3. The moduli space $\mathcal{M}_{f_{0}, \text { alg }}$ for quasihomogeneous power series w.r.t. right equivalence with $f_{0}=x^{4}+x y^{5}\left(W_{17}\right)$ is given by the variety in $\mathbf{C}^{5}$, defined by the equations

$$
Y_{4}^{2}-Y_{3} Y_{5}, Y_{3} Y_{4}-Y_{2} Y_{5}, Y_{2} Y_{4}-Y_{1} Y_{5}, Y_{3}^{2}-Y_{1} Y_{5}, Y_{2} Y_{3}-Y_{1} Y_{4}, Y_{2}^{2}-Y_{1} Y_{3}
$$

The computation of such equations is our main concern in the following section. We conclude this section by some remarks on the properties of the moduli space $\mathcal{M}_{f_{0}, \text { alg }}$.

Remark 4. (a) Since the action of $G_{f_{0}}^{\mathbf{w}}$ on $T_{-}$is given by quasihomogeneous polynomials, the quotient $T_{-} / E_{f_{0}}$ admits a $\mathbf{C}^{*}$-action. It turns out that this action is preserved by the linearization, i.e., the linearized action of $E_{f_{0}}$ commutes with the $\mathbf{C}^{*}$-action, too.

(b) Since $E_{f_{0}}$ is finite, it is reductive, so $\mathcal{M}_{f_{0}, \text { alg }}$ is an affine variety by Hilbert's Finiteness Theorem. Finiteness of $E_{f_{0}}$ implies that $\mathcal{M}_{f_{0}, \text { alg }}$ is even a geometric quotient. As an affine variety, the moduli space $\mathcal{M}_{f_{0}, \text { alg }}$ is reduced, irreducible, has the Cohen-Macaulay property, and admits a $\mathbf{C}^{*}$-action.

(c) A fine moduli space for $U n f_{f_{0}, \tau}^{-}$does not exist even if $\mathcal{S}$ is the category of complex germs (cf. [9]). Suppose $\mathcal{M}$ is a fine moduli space. Then $\mathcal{M}$ is also a coarse moduli 
space, so $\mathcal{M} \simeq T_{-} / E_{f_{0}}$. There exists a universal unfolding over the germ $\left(T_{-} / E_{f_{0}}, 0\right)$ which can be induced from the semiuniversal unfolding $F$ over the germ $\left(T_{-}, 0\right)$ and vice versa. Since $T_{-}$does not contain trivial subfamilies, the semiuniversal family $F$ over $\left(T_{-}, 0\right)$ would be universal, which contradicts Proposition 3.4.5(c).

\section{Algorithmic construction of the moduli space}

We present the main algorithm for computing equations of moduli spaces for semiquasihomogeneous power series with fixed principal part w.r.t. right equivalence, subroutines for computing the induced action of $G_{f_{0}}^{\mathbf{w}}$ on $T_{-}$by Arnold's Theorem and for a linearization of this action, and an implementation in the computer algebra system Singular 2.0 ([10]). All other subroutines are either well known algorithms in commutative algebra (cf., e.g., [19]) or are easy to implement. For a more detailed description we refer to Chapter 3 of [3].

Procedure names typed in typewriter denote built-in Singular 2.0 commands, e.g., std denotes the Standard bases algorithm of Singular 2.0.

\subsection{The action of $G_{f_{0}}^{\mathbf{w}}$}

For a quasihomogeneous polynomial $f_{0} \in \mathbf{C}\left[x_{1}, x_{2}, \ldots, x_{n}\right]$ we obtain a group action of $G_{f_{0}}^{\mathbf{w}}$ on $T_{-}$by Proposition 3.3.4, namely

$$
\begin{aligned}
G_{f_{0}}^{\mathbf{w}} \times T_{-} & \longrightarrow T_{-} \\
(\sigma, t) & \longmapsto \Theta(\sigma)(t)=s
\end{aligned}
$$

where $s \in T_{-}$is s.t. $\varphi \circ \sigma\left(F_{t}\right)=F_{s}$ for some $\varphi \in A u t_{>0}(\mathbf{C}\{\mathbf{x}\})$. We provide the algorithm ARNOLDACTION for the computation of the algebraic action of $G_{f_{0}}^{\mathbf{w}}$ on $T_{-}$ which is an implementation of the proof of Arnold's Theorem (Theorem 3.2.3). The algorithm computes the morphism $\varphi$ of order $>0$ s.t.

$$
\varphi\left(\sigma\left(F_{t}\right)\right)=F_{s}+r(\mathbf{x})
$$

where $\operatorname{ord}_{\mathbf{w}}(r)>d^{\prime \prime}:=\max \left\{|\alpha|_{\mathbf{w}}: \alpha \in B_{-}\right\}$. By Remark 3.2.1 we may omit $r(\mathbf{x})$, i.e., $\varphi\left(F_{t}\right)=F_{s}$. Since $\operatorname{ord}_{\mathbf{w}}(\varphi)>0$ Proposition 3.3 .2 implies that $s \in T_{-}$is uniquely determined by the morphism $\varphi$. It remains to compute the action $t_{i} \mapsto p_{i}(\mathbf{s}, \mathbf{t})$ by setting $p_{i}(\mathbf{s}, \mathbf{t}):=$ coefficient of $m_{i}$ in $\varphi\left(F_{t}\right)$ where $m_{1}, m_{2}, \ldots, m_{k}$ denote all upper monomials of $f_{0}$.

Algorithm ArnoldAction $\left(f_{0}, G_{f_{0}}^{\mathbf{w}}, B_{-}\right)$

In: quasihomogeneous polynomial $f_{0} \in \mathbf{K}\left[x_{1}, x_{2}, \ldots, x_{n}\right]$ with an isolated singularity at 0 , equations for the defining ideal of $G_{f_{0}}^{\mathbf{w}}$ in $\mathbf{K}\left[s_{1}, s_{2}, \ldots, s_{r}\right]$, upper monomials $B_{-}=\left\{m_{1}, m_{2}, \ldots, m_{k}\right\}$.

Out: $\left(p_{1}(\mathbf{s}, \mathbf{t}), p_{2}(\mathbf{s}, \mathbf{t}), \ldots, p_{k}(\mathbf{s}, \mathbf{t})\right)$ polynomials defining the action of $G_{f_{0}}^{\mathbf{w}}$ on $T_{-}=\mathbf{K}^{n}$. 
Note: basering has parameters $s_{1}, s_{2}, \ldots, s_{r}, t_{1}, t_{2}, \ldots, t_{k}$ and variables $x_{1}, x_{2}, \ldots, x_{n}$.

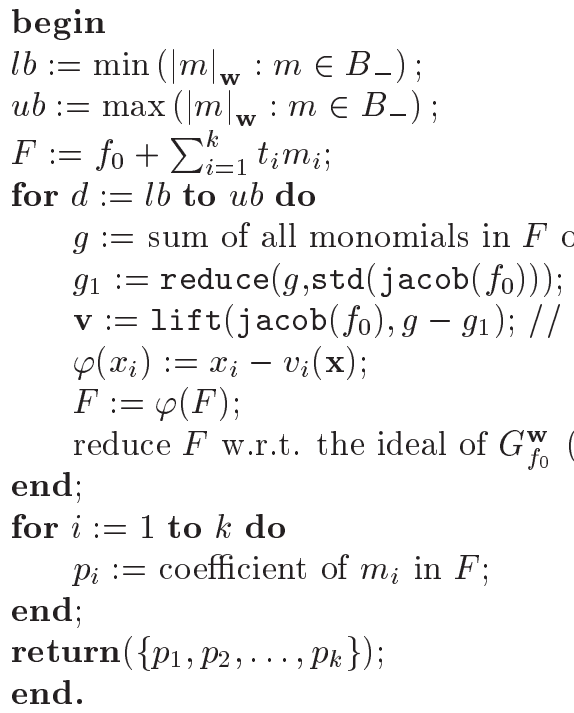

Example 4. For $f_{0}=x^{2} z+x^{2} y-z^{5}+y^{5}$ the ideal of the stabilizer $G_{f_{0}}^{(2,1,1)}$ equals $\left\langle s_{1}^{2} s_{3}^{2}-s_{3}, s_{2} s_{3}, s_{1}^{2} s_{2}+s_{1}^{2} s_{3}-1, s_{2}^{4}-s_{3}^{4}+s_{1}^{2}, s_{1}^{4}+s_{2}^{3}-s_{3}^{3}, s_{3}^{5}-s_{1}^{2} s_{3}\right\rangle$ where $G_{f_{0}}^{(2,1,1)}$ acts via the map $(x, y, z) \mapsto\left(s_{1} x, s_{3} y+s_{2} z, s_{2} y+s_{3} z\right)$, both computed by STABEQN ${ }^{3}$. The action $\alpha$ of the group $G_{f_{0}}^{(2,1,1)}$ on $T_{-}=\mathbf{C}^{3}$ is computed by ARNOLDACTION and is given by the following polynomials.

$$
\begin{aligned}
& \alpha^{*} t_{1}=p_{1}\left(\mathbf{s}, t_{1}, t_{2}, t_{3}\right)=\left(s_{3}-s_{2}\right) t_{1}, \\
& \alpha^{*} t_{2}=p_{2}\left(\mathbf{s}, t_{1}, t_{2}, t_{3}\right)=\left(s_{3}^{2}-s_{2}^{2}\right) t_{2}+2 s_{2}^{2} t_{3}^{2}, \\
& \alpha^{*} t_{3}=p_{3}\left(\mathbf{s}, t_{1}, t_{2}, t_{3}\right)=\left(s_{2}+s_{3}\right) t_{3}
\end{aligned}
$$

where $\mathbf{s}=\left(s_{1}, s_{2}, s_{3}\right)$ and the ideal of $G_{f_{0}}^{(2,1,1)}$ is contained in $\mathbf{C}[\mathbf{s}]$. In this case, the action of $G_{f_{0}}^{(2,1,1)}$ is not linear.

\subsection{Linearization of a group action}

As noted above, the algebraic action of $G_{f_{0}}^{\mathbf{w}}$ on $T_{-}$need not be linear, but this fact is irrelevant for the existence proof of the moduli space. Nonetheless, the equations of the moduli space are computed from generators of the invariant ring of the action of $G_{f_{0}}^{\mathbf{w}}$ and the corresponding algorithms only work for linear actions. Therefore we

\footnotetext{
${ }^{3}$ We refer to Section 4.3 or Ch. 3.4 of [3] for a description.
} 
need an algorithm for linearizing the action of $G_{f_{0}}^{\mathbf{w}}$ on $T_{-}$. Linearizations of algebraic actions over algebraically closed fields always exist and the construction is well known (we follow Ch. 1.6. in [4]).

Let $G$ be an algebraic group which acts algebraically on the affine variety $X$ (both defined over an algebraically closed field $\mathbf{K}$ of characteristic 0 ) via

$$
\begin{aligned}
\alpha: G \times X & \longrightarrow X \\
(\sigma, x) & \longmapsto \sigma \bullet_{\alpha} x .
\end{aligned}
$$

A linearization of $\alpha$ consists of a closed morphism $\rho: G \rightarrow G L_{n}(\mathbf{K})$ and a closed embedding $\phi: X \rightarrow \mathbf{K}^{n}$ s.t.

$$
\phi(\sigma \bullet \alpha t)=\rho(\sigma) \cdot \phi(t)
$$

where "." is the usual action of $G L_{n}(\mathbf{K})$. On the coordinate ring of $X$ we obtain the morphism $\lambda_{\sigma}(f)(x):=f\left(\alpha\left(\sigma^{-1}, x\right)\right)=\alpha^{*}(f)\left(\sigma^{-1}, x\right)$ for $\sigma \in G, f \in \mathbf{K}[X]$ and $x \in X$, which is called left-translation.

Lemma 2. Let $G$ be an algebraic group acting algebraically on a variety $X$. Any finite dimensional vector subspace $V$ of $\mathbf{K}[X]$ can be extended to a finite dimensional vector subspace $W$ of $\mathbf{K}[X]$ s.t. $G \bullet_{\alpha} W \subseteq W$. Moreover a necessary and sufficient condition that $W$ is invariant under left translation is

$$
\alpha^{*} W \subseteq \mathbf{K}[G] \otimes W
$$

Proof. Firstly, we assume $\operatorname{dim}_{\mathbf{K}}(V)=1$ and construct a $G$-invariant vectorspace $W \supseteq V$. The general case follows by constructing $G$-invariant vectorspaces for each basis element of $V$ and taking the sum of these vectorspaces.

Choose a decomposition

$$
\alpha^{*}(f)=\sum_{i=1}^{r} g_{i} \otimes h_{i} \in \mathbf{K}[G] \otimes \mathbf{K}[X]
$$

s.t. $r$ is minimal. Applying left translation to $f$ yields $\lambda_{\sigma} f(x)=\alpha^{*}(f)\left(\sigma^{-1}, x\right)=$ $\sum_{i=1}^{r} g_{i}\left(\sigma^{-1}\right) h_{i}(x)$ for all $\sigma \in G$ and $x \in X$. Hence

$$
\lambda_{\sigma}(f)=\sum_{i=1}^{r} g_{i}\left(\sigma^{-1}\right) h_{i}
$$

so $h_{1}, h_{2}, \ldots, h_{r}$ span a $\mathbf{K}$-vectorspace $W \supseteq V$ s.t. $\lambda_{\sigma}(f) \in W$ for all $\sigma \in G$. To see that $W$ is $G$-invariant take $\sigma, \tau \in G$ and choose $c_{1}, c_{2}, \ldots, c_{r} \in \mathbf{K}$ s.t. $\lambda_{\sigma^{-1} \tau}(f)=$ $\sum_{i=1}^{r} c_{i} h_{i}$. We have $\lambda_{\tau}(f)=\lambda_{\sigma \sigma^{-1} \tau}(f)=\lambda_{\sigma}\left(\sum_{i=1}^{r} c_{i} h_{i}\right)=\sum_{i=1}^{r} c_{i} \lambda_{\sigma}\left(h_{i}\right)$, so $\lambda_{\tau}(f)$ is contained in the $\mathbf{K}$-vectorspace $W_{\sigma}$ spanned by $\lambda_{\sigma}\left(h_{1}\right), \lambda_{\sigma}\left(h_{2}\right), \ldots, \lambda_{\sigma}\left(h_{r}\right)$. Since 
$r$ is minimal and $\operatorname{dim}_{\mathbf{K}}\left(W_{\sigma}\right)=r$ we have $W \cap W_{\sigma}=W$, therefore $\lambda_{\sigma}\left(h_{i}\right) \in W$ for $\sigma \in G$ and $1 \leq i \leq r$.

Now let $W \subset \mathbf{K}[X]$ be a finite-dimensional subspace and $\left\{f_{i}\right\} \cup\left\{h_{j}\right\}$ be a basis for $\mathbf{K}[X]$ s.t. $\left\{f_{i}\right\}$ spans $W$. For $f \in W$ and $\sigma \in G$ we have $\lambda_{\sigma}(f)=\sum r_{i}\left(\sigma^{-1}\right) f_{i}+$ $\sum s_{j}\left(\sigma^{-1}\right) h_{j}$, where $\alpha^{*} f=\sum r_{i} \otimes f_{i}+\sum s_{j} \otimes h_{j}$, so $\sigma \cdot f \in W$ iff $s_{j}\left(\sigma^{-1}\right)=0$ for all $\sigma \in G$. Varying $\sigma \in G$ and $f \in W$ we see that $\lambda_{\sigma}(W) \subseteq W$ iff $\alpha^{*} W \subseteq \mathbf{K}[G] \otimes W$.

Theorem 6. (Existence of equivariant embeddings) Let $G$ be an algebraic group acting algebraically on a variety $X$. There exists a finite dimensional vectorspace $V$, a closed embedding $\phi: X \hookrightarrow V$ and a morphism $\rho: G \rightarrow G L(V)$ s.t.

$$
\phi\left(\sigma \bullet_{\alpha} x\right)=\rho(\sigma) \cdot \phi(x)
$$

for all $\sigma \in G$ and $x \in X$.

Proof. By Lemma 4.2.2 we may assume that $f_{1}, f_{2}, \ldots, f_{m} \in \mathbf{K}[X]$ are algebra generators for $\mathbf{K}[X]$ which span a $G$-invariant $\mathbf{K}$-vectorspace $V$ s.t. $\alpha^{*} V \subset \mathbf{K}[G] \otimes$ $V$. Hence there exist $m_{i j} \in \mathbf{K}[G]$ s.t. $\alpha^{*} f_{i}=\sum_{j=1}^{m} m_{i j} \otimes f_{j}$ which define a map $\rho: G \rightarrow G L_{n}(\mathbf{K}), \sigma \mapsto\left(m_{i j}\left(\sigma^{-1}\right)\right)$. Note that the $m_{i j}$ 's are unique and that the map $\rho$ is a closed morphism of algebraic groups. For each $\sigma \in G$ and $x \in X$ we have

$$
f_{i}(\sigma \bullet \alpha x)=\sum_{j=1}^{m} m_{i j}\left(\sigma^{-1}\right) f_{j}(x) .
$$

Define $\phi: X \rightarrow V=\mathbf{K}^{m}$ by $y_{i}:=f_{i}(x)$ for $x \in X$, where $y_{1}, y_{2}, \ldots, y_{m}$ are coordinates on $V$. Obviously, the map $\phi$ is a morphism and, by definition, we have $\phi^{*}\left(y_{i}\right)=f_{i}$, i.e. $\phi^{*}$ is surjective, hence $\phi$ is a closed embedding of $X$. Now we can write (1) as

$$
\phi\left(\sigma \bullet_{\alpha} x\right)=\rho(\sigma) \cdot \phi(x) .
$$

In order to construct a linearization of $\alpha=\left(p_{1}, p_{2}, \ldots, p_{n}\right)$ we start with $B=$ $\left\{t_{1}, t_{2}, \ldots, t_{n}\right\}, V=\langle B\rangle$ and apply Lemma 4.2 .2 to each $t_{j}$. If some $h_{i}$ of a minimal decomposition is not contained in $V$ we add $h_{i}$ to $B$. Each such $h_{i}$ gives rise to a new component $p_{k_{i}}$ of the linearized action of $\alpha$. From a linearization $\phi: X \hookrightarrow \mathbf{K}^{m}$ of $\alpha$ and from the projection $\pi: \mathbf{K}^{m} \rightarrow \mathbf{K}^{m} / G \subset \mathbf{K}^{r}$ given by generators of the invariant ring of the linearized action of $G$ we obtain equations for the quotient of $X / G$ by computing the image of the composition $\pi \circ \phi$

$$
X \hookrightarrow \mathbf{K}^{m} \longrightarrow \mathbf{K}^{m} / G \hookrightarrow \mathbf{K}^{r} .
$$

Since $\phi$ is injective and the fibers of $\pi$ are finite (provided that $G$ is finite which is true for $G=E_{f_{0}}$ ), the map $\pi \circ \phi$ is finite and therefore it is closed. Note that this implies that the quotient $X / G$ is geometric, cf., e.g., Ch. II.3.2 in [13] (p. 96). 
The algorithm MinimalDecomposition $\left(p_{i}\right)$ returns a list $\left\{\mathbf{g}^{(i)}, \mathbf{h}^{(i)}\right\}$ s.t. $p_{i}=$ $\sum_{j=1}^{n_{i}} g_{j}^{(i)} \otimes h_{j}^{(i)} \in \mathbf{K}\left[s_{1}, s_{2}, \ldots, s_{m}\right] \otimes \mathbf{K}\left[t_{1}, t_{2}, \ldots, t_{k}\right]$ is a minimal decomposition. For a description we refer to Chapter 3.6 of [3].

Algorithm LinearizeAction $\left(G,\left\{p_{1}, p_{2}, \ldots, p_{k}\right\}\right)$

In: Ideal of $G$ in $\mathbf{K}\left[s_{1}, s_{2}, \ldots, s_{r}\right]$, polynomials $p_{1}(\mathbf{s}, \mathbf{t}), p_{2}(\mathbf{s}, \mathbf{t}), \ldots, p_{k}(\mathbf{s}, \mathbf{t})$ defining an action of $G$ on $\mathbf{K}^{k}$.

Out: $\left\{p_{1}^{\prime}(\mathbf{s}, \mathbf{t}), p_{2}^{\prime}(\mathbf{s}, \mathbf{t}), \ldots, p_{l}^{\prime}(\mathbf{s}, \mathbf{t})\right\}$, defining a linear action of $G$ on $\mathbf{K}^{l}$, $\left\{B_{1}(\mathbf{t}), B_{2}(\mathbf{t}), \ldots, B_{l}(\mathbf{t})\right\}$ an equivariant embedding $\mathbf{K}^{k} \hookrightarrow \mathbf{K}^{l}$.

begin

$B:=\left\{t_{1}, t_{2}, \ldots, t_{k}\right\} ; l:=k$

$\left(p_{1}^{\prime}, p_{2}^{\prime}, \ldots, p_{k}^{\prime}\right):=p_{1}, p_{2}, \ldots, p_{k}$;

for $i:=1$ to $k$ do

$\left\{\mathbf{g}^{(i)}, \mathbf{h}^{(i)}\right\}:=$ MinimalDecomposition $\left(p_{i}\right) ;$

for $j:=1$ to $n_{i}$ do

if $h_{j}^{(i)} \notin\langle B\rangle_{\mathrm{K}}$ then begin

$B:=B \cup\left\{h_{j}^{(i)}\right\} ; l=l+1 ; / /$ extend basis

$p_{l}^{\prime}:=h_{j}^{(i)}\left(p_{1}, p_{2}, \ldots, p_{k}\right) ; / /$ new component of the action

replace $h_{j}^{(i)}$ in $p_{1}^{\prime}, p_{2}^{\prime}, \ldots, p_{l}^{\prime}$ by the new variable $t_{l}$;

\section{end if}

end

end

$\operatorname{return}\left(\left\{p_{1}^{\prime}, p_{2}^{\prime}, \ldots, p_{l}^{\prime}\right\}, B\right)$

end.

We continue with our running example in order to illustrate the algorithm.

Example 5. Let $f_{0}=x^{2} z+x^{2} y-z^{5}+y^{5}$. We have to extend the $\mathbf{C}$-vectorspace $V$, spanned by $\left\langle t_{1}, t_{2}, t_{3}\right\rangle_{\mathbf{C}}$, to a $G_{f_{0}}^{\mathbf{w}}$ - invariant vectorspace $W$. The decomposition of the nonlinear component $p_{2}=\left(s_{3}^{2}-s_{2}^{2}\right) t_{2}+2 s_{2}^{2} t_{3}^{2}$ of $\alpha=\left(p_{1}, p_{2}, p_{3}\right)$ equals

$$
\alpha^{*} t_{2}=p_{2}\left(s_{1}, s_{2}, s_{3}, t_{1}, t_{2}, t_{3}\right)=\left(s_{3}^{2}-s_{2}^{2}\right) \otimes t_{2}+2 s_{2}^{2} \otimes t_{3}^{2} .
$$

Therefore we consider the vectorspace $W:=\left\langle t_{1}, t_{2}, t_{3}, t_{3}^{2}\right\rangle_{\mathbf{C}}$ and we set $t_{4}=t_{3}^{2}$ and $\operatorname{deg}\left(t_{4}\right)=2$. The new component of the action is given by

$$
\alpha^{*} t_{4}=\alpha^{*} t_{3}^{2}=\left(s_{2}+s_{3}\right)^{2} t_{3}^{2}=\left(s_{2}^{2}+2 s_{2} s_{3}+s_{3}^{2}\right) t_{4}=\left(s_{2}^{2}+s_{3}^{2}\right) t_{4}
$$

since $s_{2} s_{3}$ is in the ideal of $G_{f_{0}}^{\mathbf{w}}$. Hence $W$ is the desired $G_{f_{0}}^{\mathbf{w}}$-invariant vectorspace. The closed embedding $\phi: T_{-} \hookrightarrow \mathbf{C}^{4}$ is given by

$$
\left(t_{1}, t_{2}, t_{3}\right) \longmapsto\left(t_{1}, t_{2}, t_{3}, t_{3}^{2}\right)
$$


and the representation $\rho: G_{f_{0}}^{\mathbf{w}} \rightarrow G L_{4}(\mathbf{C})$ by

$$
\left(s_{1}, s_{2}, s_{3}\right) \longmapsto\left(\begin{array}{cccc}
s_{3}-s_{2} & 0 & 0 & 0 \\
0 & s_{3}^{2}-s_{2}^{2} & 0 & 2 s_{2}^{2} \\
0 & 0 & s_{2}+s_{3} & 0 \\
0 & 0 & 0 & s_{2}^{2}+s_{3}^{2}
\end{array}\right)
$$

The ideal of the variety $\phi\left(T_{-}\right)$is generated by $t_{4}-t_{3}^{2}$ and the generator is quasihomogeneous of type $(2 ; 1,2,1,2)$. The group $G_{f_{0}}^{\mathbf{w}}$ acts linearly but not faithfully on $\phi\left(T_{-}\right)$. The ideal of the group $E_{f_{0}}^{\prime}:=\rho\left(G_{f_{0}}^{\mathbf{w}}\right)$ computed by ImaGEGrouP ${ }^{4}$ is

$$
\mathcal{I}\left(E_{f_{0}}^{\prime}\right)=\left\langle s_{5}^{5}-1, s_{4}^{2}-s_{5}, s_{3}+s_{4} s_{5}^{3}-s_{5}, s_{2}+s_{3}-s_{5}, s_{1}-s_{5}^{3}\right\rangle
$$

and may be simplified ${ }^{5}$ to $\mathcal{I}\left(E_{f_{0}}^{\prime}\right)=\left\langle s_{4}^{10}-1\right\rangle$ so the linear action on $\phi\left(T_{-}\right)$(and $\left.\mathbf{C}^{4}\right)$ is given by

$$
\left(s_{4}, t_{1}, t_{2}, t_{3}, t_{4}\right) \longmapsto\left(s_{4}^{6} t_{1}, s_{4}^{7} t_{2}-s_{4}^{7} t_{4}+s_{4}^{2} t_{4}, s_{4} t_{3}, s_{4}^{2} t_{4}\right) .
$$

Note that $\left|G_{f_{0}}^{\mathbf{w}}\right|=20$ and $\left|E_{f_{0}}^{\prime}\right|=10$.

The algorithm LinEARIZEACTION preserves $\mathbf{C}^{*}-$ actions.

Proposition 7. Let $\alpha$ be an action of $G$ on $\mathbf{C}^{k}$ which commutes with a $\mathbf{C}^{*}$-action and let $\mathbf{w}$ be s.t. the defining polynomials of $\alpha$ are quasihomogeneous w.r.t. $\mathbf{w}$, where $t_{1}, t_{2}, \ldots, t_{k}$ are coordinates on $\mathbf{C}^{k}$ and the variables $s_{1}, s_{2}, \ldots, s_{r}$ of $\mathbf{C}[G]$ have weight 0 . The linearized action, constructed by LINEARIZEACTION, commutes with a $\mathbf{C}^{*}$-action, i.e., the polynomials $p_{1}(\mathbf{s}, \mathbf{t}), p_{2}(\mathbf{s}, \mathbf{t}), \ldots, p_{l}(\mathbf{s}, \mathbf{t})$ are quasihomogeneous w.r.t. to the extended weight $\mathbf{w}^{\prime}$ where $\operatorname{deg}_{\mathbf{w}^{\prime}}\left(t_{i}\right)=\operatorname{deg}_{\mathbf{w}}\left(t_{j}\right)$ if $i>k$ and $t_{i}$ comes from the decomposition of $\alpha^{*} t_{i}$. In particular, the ideal of $\phi\left(T_{-}\right)$is quasihomogeneous w.r.t. the weights of $t_{1}, t_{2}, \ldots, t_{l}$.

Proof. We refer to Proposition 3.6.69 of [3] (p. 56).

\subsection{Computation of equations for the moduli space}

Given a semiquasihomogeneous polynomial $f_{0}$ of type $(d ; \mathbf{w})$ we compute equations for a coarse moduli space of semiquasihomogeneous power series with principal part $f_{0}$ as follows. Firstly, we compute equations for the stabilizer $G_{f_{0}}^{\mathbf{w}}$ by means of StabEQn and upper monomials for $M_{f_{0}}$ with UpPerMonomials (see below). Then ArnoldAction yields an algebraic action $\alpha$ of $G_{f_{0}}^{\mathbf{w}}$ on $T_{-}$which is linearized by $(\phi, \rho)$, obtained from LinEARIZEACTION (note that an action yields a representation). Equations for $E_{f_{0}}^{\prime}$, the image of $G_{f_{0}}^{\mathbf{w}}$ under $\rho$, are computed and QuoTIENTEQuATIOns (based on Derksen's Algorithm, cf. [5], and IMAGEVARIETY) provides

\footnotetext{
${ }^{4}$ The resulting equations may contain more variables than the equations of the input.

${ }^{5}$ Since the simplification is done by elimpart (cf. [8]) and by hand, it is not contained in the current version of qhmoduli.lib.
} 
equations for an embedding of the moduli space. Below we give a brief description of the algorithms UpperMonomials, StabEqn, ImageVAriety, ImageGroup and QuotientEquations. For a thorough description we refer to Chapter 3 in [3].

1. Uppermonomials: Computes the set $\left\{m_{1}, m_{2}, \ldots, m_{k}\right\}$ of upper monomials of a monomial basis of the Milnor algebra $\mathbf{C}\left\{x_{1}, x_{2}, \ldots, x_{n}\right\} / j\left(f_{0}\right)$ of $f_{0}$. This requires a standard basis computation in a local ring.

2. StabEqn: Computes equations of the stabilizer group $G_{f_{0}}^{\mathbf{w}} \subset G^{\mathbf{w}} \subset \operatorname{Aut}(\mathbf{C}\{\mathbf{x}\})$ of $f_{0}$ by comparing coefficients of $f_{0}$ and $\sigma\left(f_{0}\right)$ where the elements $\sigma \in G^{\mathbf{w}}$ satisfy $\sigma\left(x_{i}\right)=\sum_{\langle\mathbf{w}, \alpha\rangle=w_{i}} s_{i, \alpha} \mathbf{x}^{\alpha}, \operatorname{det}\left(\frac{\partial \sigma\left(x_{i}\right)}{\partial x_{j}}\right) \neq 0$ and $s_{i, \alpha}$ are new indeterminates. The equations are simplified by using the command elimpart from the Singular 2.0 library presolve.lib, cf. [8]. Since $f_{0}$ has an isolated singularity at 0 the zeroset of the resulting ideal is a group. This step needs the command radical.

3. ImageVariety and ImageGroup: Computes the ideal $I$ of the image of $T_{-}$ under the morphism

$$
\Phi: T_{-} \hookrightarrow \mathbf{C}^{l} \rightarrow \mathbf{C}^{l} / E_{f_{0}}^{\prime} \hookrightarrow \mathbf{C}^{m}
$$

given by $\Phi^{*}\left(y_{i}\right)=h_{i}$. Since $h_{1}, h_{2}, \ldots, h_{m}$ are quasihomogeneous $\left(\mathbf{C}^{l} / E_{f_{0}}^{\prime}\right.$ admits a $\mathbf{C}^{*}$-action) we apply the Hilbert-driven standard bases algorithm (stdhilb), which proves to be much more efficient in our application (cf. [18]). The coarse moduli space for $U n f_{f_{0}}^{-}$is given by the variety $\mathcal{V}(I)$, defined by the ideal $I$. In particular, as sets we have

$$
\mathcal{V}(I) \simeq U n f_{f_{0}}^{-}(\{p t\}) .
$$

IMAGEGROUP computes equations of $E_{f_{0}}^{\prime}$ from the equations of $G_{f_{0}}^{\mathbf{w}}$ and the linearized polynomials $p_{1}^{\prime}, p_{2}^{\prime}, \ldots, p_{l}^{\prime}$ of the action of $G_{f_{0}}^{\mathbf{w}}$. May introduce additional variables.

4. QuotientEquations: Given equations of an algebraic group $G$, of a variety $X$, and polynomials defining a linear action of $G$ on $X$, QUoTIEnTEQUATIONS computes equations of an embedding of the quotient $X / G$. Generators of the invariant ring $\mathbf{C}\left[t_{1}, t_{2}, \ldots, t_{l}\right]^{G}$ are computed by Derksen's algorithm ([5]), which requires equations for $G$ and polynomials defining the action. In general, one has to apply the Reynolds operator, in which case $G$ must be finite. In the implementation one can choose to use equations for $G_{f_{0}}^{\mathbf{w}}$ or $E_{f_{0}}^{\prime}$ to compute the invariant ring w.r.t. the linearized action (running times may be different).

In the algorithm the group $G_{f_{0}}^{\mathbf{w}}$ is described by its defining ideal and the action of $G_{f_{0}}^{\mathbf{w}}$ on $T_{-}$is represented by polynomials $p_{1}(\mathbf{s}, \mathbf{t}), \ldots, p_{k}(\mathbf{s}, \mathbf{t})$ where $\mathbf{s}=\left(s_{1}, s_{2}, \ldots, s_{r}\right)$, $\mathbf{t}=\left(t_{1}, t_{2}, \ldots, t_{k}\right)$. The linearized action is represented by the polynomials 
$p_{1}\left(\mathbf{s}, \mathbf{t}^{\prime}\right), \ldots, p_{l}\left(\mathbf{s}, \mathbf{t}^{\prime}\right)$ where $l \geq k$ and $\mathbf{t}^{\prime}=\left(t_{1}, t_{2}, \ldots, t_{l}\right)$, which also encode the representation $\rho$. From a theoretical point of view, the computation of $E_{f_{0}}^{\prime}$ is not necessary, but this may simplify the computation of the Reynolds operator since $\left|E_{f_{0}}^{\prime}\right| \leq\left|G_{f_{0}}^{\mathbf{w}}\right|$. The change of the basering, which occurs at several occasions in the Singular 2.0 implementation, is omitted.

Algorithm ModEQN $\left(f_{0}\right)$

In: quasihomogeneous polynomial $f_{0}$ with an isolated singularity at 0

Out: equations $f_{1}, f_{2}, \ldots, f_{q}$ of the moduli space of $U n f_{f_{0}}^{-}$.

begin

$\mathbf{m}:=\operatorname{UpperMonomials}\left(f_{0}\right) ; / /$

$k:=\operatorname{size}(\mathbf{m})$;

$G_{f_{0}}^{\mathbf{w}}:=\operatorname{STABEQN}\left(f_{0}\right) ; / /$ variables $s_{1}, s_{2}, \ldots, s_{r}$

$/ /$ variables are $s_{1}, s_{2}, \ldots, s_{r}, t_{1}, t_{2}, \ldots, t_{k}, x_{1}, x_{2}, \ldots, x_{n}$

$F_{t}:=f_{0}+\sum_{i=1}^{k} t_{i} \mathbf{m}[i]$

$\left\{p_{1}(\mathbf{s}, \mathbf{t}), \ldots, p_{k}(\mathbf{s}, \mathbf{t})\right\}:=\operatorname{ArnoldaAction}\left(G_{f_{0}}^{\mathbf{w}}, F_{t}\right) ;$

$\left(\left\{p_{1}^{\prime}\left(\mathbf{s}, \mathbf{t}^{\prime}\right), \ldots, p_{l}^{\prime}\left(\mathbf{s}, \mathbf{t}^{\prime}\right)\right\}, \phi\right):=\operatorname{LinEARIZEACTION}\left(G_{f_{0}}^{\mathbf{w}},\left\{p_{1}, p_{2}, \ldots, p_{k}\right\}\right)$;

$/ /$ variables are $s_{1}, s_{2}, \ldots, s_{r}, t_{1}, t_{2}, \ldots, t_{k}, t_{k+1}, \ldots, t_{l}$

$E_{f_{0}}^{\prime}:=\operatorname{ImageGroup}\left(G_{f_{0}}^{\mathbf{w}}, p_{1}^{\prime}, p_{2}^{\prime}, \ldots, p_{l}^{\prime}\right)$;

$\left\{g_{1}, g_{2}, \ldots, g_{l}\right\}:=\left\{\phi_{1}\left(t_{1}, \ldots, t_{k}\right), \phi_{2}\left(t_{1}, \ldots, t_{k}\right), \ldots, \phi_{l}\left(t_{1}, \ldots, t_{k}\right)\right\} ;$

// variables are $s_{1}, s_{2}, \ldots, s_{r^{\prime}}, t_{1}, t_{2}, \ldots, t_{k}, t_{k+1}, \ldots, t_{l}$;

$\left\{g_{1}^{\prime}, g_{2}^{\prime}, \ldots, g_{l^{\prime}}^{\prime}\right\}:=\operatorname{IMAGEVARIETy}\left(\{0\},\left\{g_{1}, g_{2}, \ldots, g_{l}\right\}\right)$;

// IMAGEVARIETy provides the ideal of the embedding of $T_{-}$

$\left\{f_{1}, \ldots, f_{q}\right\}:=$ QuotientEQuations $\left(E_{f_{0}}^{\prime},\left\{p_{1}^{\prime}, p_{2}^{\prime}, \ldots, p_{l}^{\prime}\right\},\left\{g_{1}^{\prime}, \ldots, g_{l^{\prime}}^{\prime}\right\}\right)$;

end;

$\operatorname{return}\left(\left\{f_{1}, f_{2}, \ldots, f_{q}\right\}\right)$;

\subsection{Singular 2.0 Implementation}

All algorithms from the previous section are implemented in the Singular 2.0 libraries qhmoduli.lib,rinvar.lib and zeroset.lib. A short description is given below, a full description for all algorithms and their options is contained in [3].

1. qhmoduli.lib: Contains the main algorithms for computing equations for moduli spaces for semiquasihomogeneous power series with fixed principal part (e.g., ModEqn, ArnoldAction and StabEqn).

2. rinvar.lib: Contains algorithms for computing invariant rings of reductive groups which are given by equations and algebraic actions (i.e., defined by polynomials). The computation of the invariant ring is based on Derksen's algorithm (NullCone), cf. [5]. Note that the Reynolds operator is implemented for finite groups only. 
3. zeroset.lib: Contains algorithms for computing the zeroset of a zero-dimensional ideal (ZEROSET) and factorization of univariate polynomials (FACTOR) over finite extensions of $\mathbf{Q}$.

4.4.1. The example $f_{0}=x^{2} z+x^{2} y-z^{5}+y^{5}$

We show how to compute the moduli space of semiquasihomogeneous hypersurface singularities w.r.t. the fixed part $f_{0}=x^{2} z+x^{2} y-z^{5}+y^{5}$ by means of the SingulaR 2.0 library qhmoduli.lib.

$>$ LIB ' 'qhmoduli.lib', ;

$>\operatorname{ring} \mathrm{R}=0,(\mathrm{x}, \mathrm{y}, \mathrm{z}), \mathrm{ls}$;

$>$ poly $f=-z 5+y 5+x 2 z+x 2 y$;

$>$ list $\operatorname{stab}=\operatorname{StabEqn}(f) ;>\operatorname{def} \mathrm{R} 1=\operatorname{stab}[1]$; setring $\mathrm{R} 1$;

$>$ stabid;

stabid $[1]=s(2) * s(3)$

stabid $[2]=s(1)-2 * s(2)+s(1)-2 * s(3)-1$

stabid $[3]=s(1)-2 * s(3)-2-s(3)$

stabid $[4]=s(2)-4-s(3) \wedge 4+s(1) \wedge 2$

stabid $[5]=s(1)-4+s(2) \wedge 3-s(3)-3$

stabid $[6]=s(3)-5-s(1)-2 * s(3)$

$>\operatorname{vdim}($ stabid) $; 20$

$>$ setring $R$;

$>$ StabOrder $(f)$;

20

$>$ ideal $\mathrm{B}=$ UpperMonomials $(\mathrm{f})$;

$>\mathrm{B}$;

$\mathrm{B}[1]=\mathrm{y} 3 \mathrm{z} 3$

$B[2]=x 2 y 3$

$\mathrm{B}[3]=\mathrm{x} 2 \mathrm{y} 2$

$>$ def R2 = ArnoldAction ( $f$, stab,B);

$>$ setring $\mathrm{R} 2$;

$>$ actionid;

actionid $[1]=-\mathrm{s}(2) * \mathrm{t}(1)+\mathrm{s}(3) * \mathrm{t}(1)$

actionid [2] =-s (2) ^ $2 * \mathrm{t}(2)+2 * \mathrm{~s}(2)-2 * \mathrm{t}(3) \sim 2+\mathrm{s}(3)-2 * \mathrm{t}(2)$

actionid $[3]=\mathrm{s}(2) * \mathrm{t}(3)+\mathrm{s}(3) * \mathrm{t}(3)$

$>\operatorname{nvars}(\operatorname{stab}[1])$;

3

$>\operatorname{def} \mathrm{R} 3=\operatorname{LinearizeAction}($ stabid, $\operatorname{actionid,nvars}(\operatorname{stab}[1])$ );

$>$ setring $\mathrm{R} 3$;

$>$ actionid;

actionid $[1]=-\mathrm{s}(2) * \mathrm{t}(1)+\mathrm{s}(3) * \mathrm{t}(1)$

actionid $[2]=-\mathrm{s}(2) \sim 2 * \mathrm{t}(2)+2 * \mathrm{~s}(2) \sim 2 * \mathrm{t}(4)+\mathrm{s}(3) \sim 2 * \mathrm{t}(2)$ 


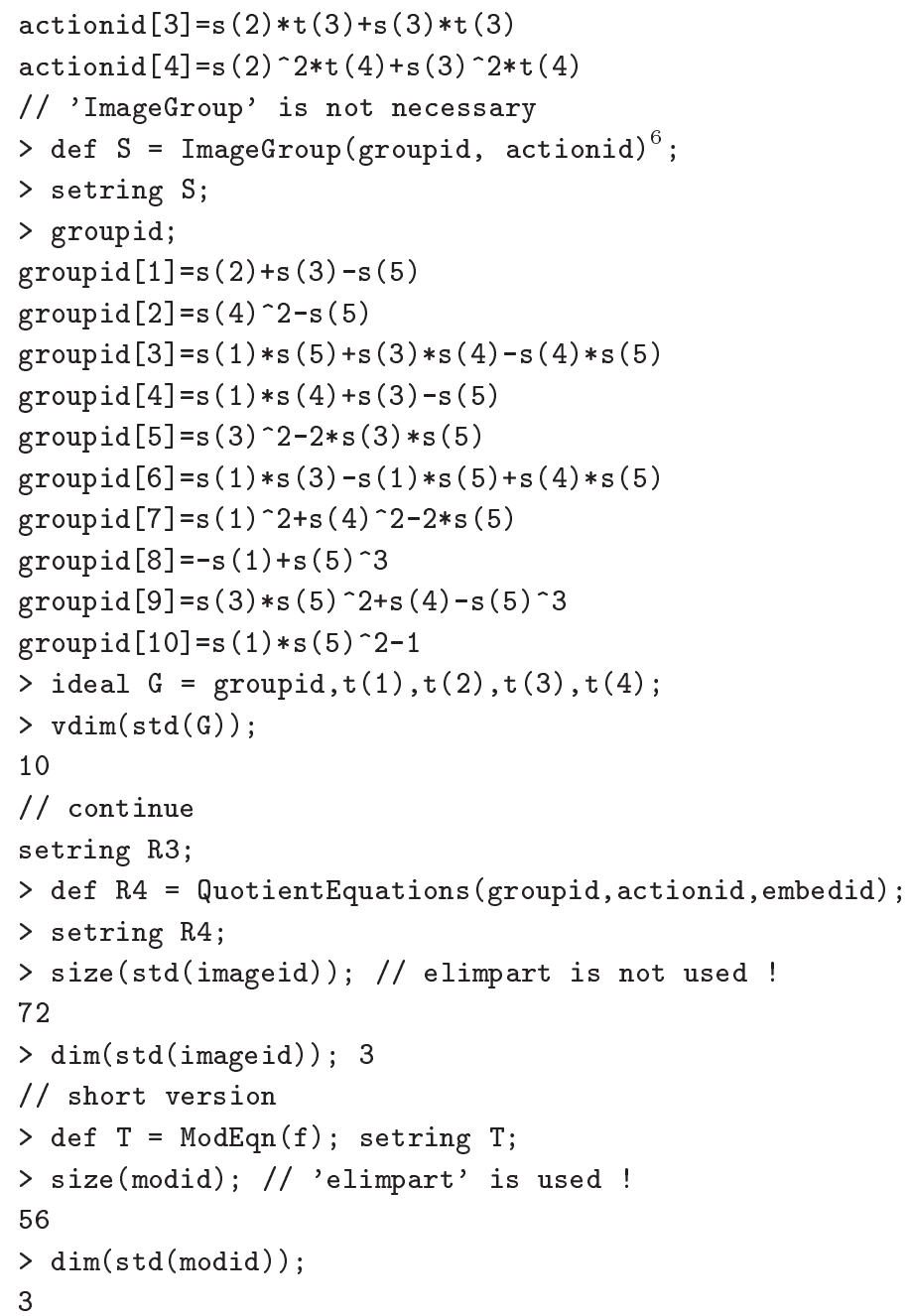

\subsubsection{Performance}

We provide 6 examples with increasing complexity and runtime. The first table contains information about the singularity, the group action and the invariant ring together with the embedding of the moduli space while the second table contains a profile of the computation. We consider the following examples

\footnotetext{
${ }^{6}$ Recall that the algorithm may introduce additional variables to those contained in groupid.
} 


\begin{tabular}{|l|l|l|l|l|}
\hline 1. & $\mathrm{E}_{19}$ & $x y^{7}+x^{3}$ & 4. & $x^{2} y+x^{2} z+y^{5}-z^{5}$ \\
\hline 2. & $\mathrm{~V}_{19}^{* 1}$ & $x^{2} y+y^{4} z+z^{4}$ & 5. & $x^{5} y-x y^{5}$ \\
\hline 3. & $\mathrm{Z}_{23}$ & $y^{11}+x^{3} y$ & 6. & $x^{8}+14 x^{4} y^{4}+y^{8}$ \\
\hline
\end{tabular}

where examples 1, 2, 3 are taken from [20]. Note that stabilizers of Example 3,4,5 contain matrices with more than one entry per row. A "?" denotes that the computation was aborted due to time or memory constraints (more than $12 \mathrm{~h}$ or $700 \mathrm{MB}$ ). For more examples we refer to Chapter 4.2 in [3] (p. 70ff).

\begin{tabular}{|r|r|r|r|r|r|r|r|r|}
\hline Nr. & $\mu$ & $t_{-}$ & $\left|G_{f_{0}}^{\mathbf{w}}\right|$ & $\left|E_{f_{0}}^{\prime}\right|$ & $l$ & $\# I$ & $\# E$ & $u b$ \\
\hline 1 & 19 & 2 & 21 & 21 & 2 & 8 & 21 & 21 \\
\hline 2 & 19 & 3 & 32 & 16 & 3 & 22 & 191 & 16 \\
\hline 3 & 23 & 3 & 33 & 33 & 3 & 35 & 673 & 33 \\
\hline 4 & 24 & 3 & 20 & 10 & 4 & 21 & 56 & 10 \\
\hline 5 & 25 & 3 & 144 & $?$ & 3 & $?$ & $?$ & $?$ \\
\hline 6 & 49 & 10 & 192 & $?$ & $>10$ & $?$ & $?$ & $?$ \\
\hline
\end{tabular}

Here $\mu$ denotes the Milnor number, $t_{-}$the dimension of $T_{-}, l$ is the dimension of the ambient space of the embedding of $T_{-}$via $\phi, \# I, \# E$ are the numbers of generators for the invariant ring $\mathbf{C}\left[t_{1}, t_{2}, \ldots, t_{l}\right]^{E_{f_{0}}^{\prime}}$ respectively ideal of the quotient (deg-lex. Gröbner basis) and $u b$ denotes highest degree occurring in the basis of the invariant ring. Note that $\operatorname{dim} T_{-}=l$ implies $\phi=i d$.

In the table below we provide running times (measured in seconds) for these examples computed in Singular-2-0-0 running under Linux on a $1 \mathrm{GHz}$ Athlon with $768 \mathrm{MB}$ RAM. Here we denote by $G_{f_{0}}^{\mathbf{w}}$ the time for STABEQN, by $\Theta$ the time for ARNoldACTion, by $\rho$ the time for LinEARIzEAction, by $E_{f_{0}}^{\prime}$ the time for ImageGroup, by NC the time for NullCone, by R1, R2 the timer for computing the Reynolds operator, resp. applying the operator to all non-invariant generators, and by Eq the time for IMAGEIDEAL. In the last column we provide the running time of ModEQN.

\begin{tabular}{|r|r|r|r|r|r|r|r|r|r|}
\hline Nr. & $G_{f_{0}}^{\mathbf{w}}$ & $\Theta$ & $\rho$ & $E_{f_{0}}^{\prime}$ & $\mathrm{NC}$ & $\mathrm{R} 1$ & $\mathrm{R} 2$ & $\mathrm{Eq}$. & tot. \\
\hline 1 & 0.02 & 0.02 & 0.00 & 0.01 & 0.02 & 0 & 0 & 0.07 & 0.13 \\
\hline 2 & 0.03 & 0.05 & 0.00 & 0.01 & 0.06 & 0 & 0 & 15.46 & 15.60 \\
\hline 3 & 0.02 & 0.04 & 0.01 & 0.15 & 0.00 & 0 & 0 & 829.35 & 829.56 \\
\hline 4 & 0.05 & 0.15 & 0.04 & 0.01 & 0.16 & 0.90 & 0.00 & 4.80 & 5.10 \\
\hline 5 & 0.03 & 0.37 & 0.01 & $?$ & $?$ & $?$ & $?$ & $?$ & $?$ \\
\hline 6 & 0.04 & 6910.00 & $?$ & $?$ & $?$ & $?$ & $?$ & $?$ & $?$ \\
\hline
\end{tabular}

\section{Brieskorn-Pham singularities}

A polynomial $f_{0} \in \mathbf{C}\left[x_{1}, x_{2}, \ldots, x_{n}\right]$ is called a Brieskorn-Pham polynomial if $f_{0}=$ $\sum_{i=1}^{n} x_{i}^{a_{i}}$ for $a_{1}, a_{2}, \ldots, a_{n} \in \mathbf{N}$. Brieskorn-Pham polynomials appeared, e.g., in the study of exotic spheres pursued by Milnor, Brieskorn and others in the 1960's. For 
semiquasihomogeneous power series with principal part a Brieskorn-Pham polynomial the computation of the moduli space may be much simpler because of the following two facts :

- Generators for $G_{f_{0}}^{\mathbf{w}}$ can be written down without computation.

- The image of $\Theta$ is contained in $G L_{k}(\mathbf{C})$, i.e., the induced group action of $G_{f_{0}}^{\mathbf{w}}$ on $T_{-}$is linear.

Hence one may compute all elements of $G_{f_{0}}^{\mathbf{w}}$ or $E_{f_{0}}$, which are needed to compute the Reynolds operator, by matrix multiplication instead of computing the zeroset of a 0 -dim. ideal (the ideal of $G_{f_{0}}^{\mathbf{w}}$ or $E_{f_{0}}$ ). In general, the Reynolds operator must be used since Derksen's algorithm computes generators for the ideal generated by $\mathbf{C}\left[t_{1}, t_{2}, \ldots, t_{m}\right]_{+}^{G_{f_{0}}^{\mathbf{w}}}$, which are not necessarily invariant. This might be a significant improvement in the computation, cf., e.g., Example 5.7.

In order to prove the structure theorem on $G_{f_{0}}^{\mathbf{w}}$ we need the following Lemma, which was published (without proof) in 1991 by Orlik and Wagreich.

Lemma 3. [15] Let $f \in \mathbf{C}\left[x_{1}, x_{2}, \ldots, x_{n}\right]$ be quasihomogeneous and $G=G_{f_{0}}^{\mathbf{w}} \cap$ $G L_{n}(\mathbf{C})$. Then

$$
\bigoplus_{i=1}^{n} \mathbf{C} \frac{\partial f}{\partial x_{i}}
$$

is a $G$-module.

Proof. For $\sigma \in G_{f_{0}}^{\mathbf{w}} \cap G L_{n}(\mathbf{C})$ we have $f_{0}=f_{0} \circ \sigma$ (composition of functions) and therefore $\frac{\partial f_{0}}{\partial x_{i}}(x)=\frac{\partial f_{0} \circ \sigma}{\partial x_{i}}(x)=\sum_{j=1}^{n} \frac{\partial f_{0}}{\partial x_{j}}(\sigma \cdot x) \frac{\partial \sigma_{j}}{\partial x_{i}}(x)$. Since $\sigma \in G L_{n}(\mathbf{C})$ the terms $\frac{\partial \sigma_{j}}{\partial x_{i}}(x)$ are constants which we denote by $\lambda_{j} \in \mathbf{C}$. We obtain $\frac{\partial f_{0}}{\partial x_{i}}(x)=$ $\left(\sum_{j=1}^{n} \lambda_{j} \frac{\partial f_{0}}{\partial x_{j}}\right)(\sigma \cdot x)$. Now

$$
\begin{aligned}
\sigma \cdot \frac{\partial f_{0}}{\partial x_{i}}(x) & =\sigma \cdot\left(\sum_{j=1}^{n} \lambda_{j} \frac{\partial f_{0}}{\partial x_{j}}\right)(\sigma \cdot x)=\left(\sum_{j=1}^{n} \lambda_{j} \frac{\partial f_{0}}{\partial x_{j}}\right)\left(\sigma^{-1} \sigma \cdot x\right) \\
& =\sum_{j=1}^{n} \lambda_{j} \frac{\partial f_{0}}{\partial x_{j}}(x) .
\end{aligned}
$$

By using the previous lemma we obtain a theorem about the structure of the stabilizer groups of Brieskorn-Pham polynomials.

Theorem 7. Let $f_{0} \in \mathbf{C}\left[x_{1}, x_{2}, \ldots, x_{n}\right]$ be a Brieskorn-Pham polynomial.

(a) For $f_{0}=\sum_{i=1}^{n} x_{i}^{d}$ the group $G_{f_{0}}^{\mathbf{w}}$ is generated by the diagonal matrix containing precisely one $d$-th root of 1 and all $n \times n$ permutation matrices, provided that $d>2$. 
(b) Let $f_{0}=\sum_{i=1}^{m} f_{0}^{\left(d_{i}\right)}$ where $f_{0}^{\left(d_{i}\right)}=\sum_{j=r_{i-1}+1}^{r_{i}} x_{j}^{d_{i}}$ s.t. $d_{i}, r_{i} \in \mathbf{N}, 0<d_{i}<d_{j}$ for $i<j$ and $r_{0}=0, r_{i}<r_{i+1}$. The stabilizer of $f_{0}$ is

$$
G_{f_{0}}^{\mathbf{w}}=\bigoplus_{i=1}^{m} G_{f_{0}^{\left(d_{i}\right)}}^{\mathbf{w}}
$$

Proof. (a) Since all weights are equal (to 1 ) the only quasihomogeneous automorphisms are matrices, i.e., $G_{f_{0}}^{\mathbf{w}} \subset G L_{n}(\mathbf{C})$. Let $M=\bigoplus_{i=1}^{n} \mathbf{C} \frac{\partial f_{0}}{\partial x_{i}}=\mathbf{C} x_{1}^{d-1} \oplus \mathbf{C} x_{2}^{d-1} \oplus$ $\ldots \oplus \mathbf{C} x_{n}^{d-1}$ and assume w.l.o.g. that $A=\left(a_{i j}\right) \in G_{f_{0}}^{\mathbf{w}}$ is s.t. $a_{11}$ and $a_{12}$ are not equal to 0. By Lemma $5.3 M$ is a $G_{f_{0}}^{\mathbf{w}}-$ module, but $A^{-1} \cdot x_{1}^{d-1}$ contains terms like $a_{11}^{d-2} a_{12} x_{1}^{d-2} x_{2}$, and is therefore not contained in $M$, a contradiction. Hence any matrix in $G_{f_{0}}^{\mathbf{w}}$ contains precisely one nonzero entry per row, which must be a $d$-th root of unity. Since $f_{0}$ is invariant under permutations the claim follows.

(b) By assumption we have $\operatorname{deg}_{\mathbf{w}}\left(x_{1}\right)=\operatorname{deg}_{\mathbf{w}}\left(x_{2}\right)=\ldots=\operatorname{deg}_{\mathbf{w}}\left(x_{r_{1}}\right)>\operatorname{deg}_{\mathbf{w}}\left(x_{r_{1}+j}\right)$ and for $r_{i-1}<k \leq r_{i}$ each automorphism $\varphi \in G_{f_{0}}^{\mathbf{w}}$ is of the form

$$
\varphi\left(x_{k}\right)=\sum_{j=r_{i-1}+1}^{r_{i}} \lambda_{k j} x_{j}+c_{k} R_{k}\left(x_{r_{i}+1}, x_{r_{i}+2}, \ldots, x_{n}\right)
$$

for some $\lambda_{k j}, c_{k} \in \mathbf{C}$ and $R_{k} \in \mathbf{C}\left[x_{r_{i}+1}, x_{r_{i}+2}, \ldots, x_{n}\right]$ with $\operatorname{deg}_{\mathbf{w}}\left(R_{k}\right)=w_{k}$ s.t. $\operatorname{det}\left(\frac{\partial \varphi}{\partial x_{i}}(0)\right) \neq 0$. We show that $c_{k}=0$ and that the linear part of $\varphi$ satisfies part (a) for each block $x_{r_{i-1}+1}, x_{r_{i-1}+2}, \ldots, x_{r_{i}}$ of variables which proves the claim. Let $i$ be minimal s.t. $\varphi\left(x_{k}\right)$ is not as required for some $r_{i-1}<k \leq r_{i}$ and define $\varphi^{\prime}\left(x_{k}\right)=x_{k}$ for $1 \leq k \leq r_{i-1}, \varphi^{\prime}\left(x_{k}\right)=\sum \lambda_{k j} x_{j}$ for $r_{i-1}<k \leq r_{i}$ and $\varphi^{\prime}\left(x_{k}\right)=\varphi\left(x_{k}\right)$ otherwise. By assumption, $\varphi\left(x_{1}\right), \varphi\left(x_{2}\right), \ldots, \varphi\left(x_{r_{i-1}}\right)$ only affect $x_{1}, x_{2}, \ldots, x_{r_{i-1}}$ which cannot be changed by $\varphi\left(x_{r_{i-1}+j}\right)$ for $j>0$ and $x_{r_{i-1}+1}, x_{r_{i-1}+2}, \ldots, x_{r_{i}}$ are affected by $\varphi\left(x_{r_{i-1}+1}\right), \varphi\left(x_{r_{i-1}+2}\right), \ldots, \varphi\left(x_{r_{i}}\right)$. Note that $\varphi\left(f_{0}^{\left(d_{i}\right)}+f_{0}^{\left(d_{i+1}\right)}+\ldots+f_{0}^{\left(d_{m}\right)}\right)=f_{0}^{\left(d_{i}\right)}+$ $f_{0}^{\left(d_{i+1}\right)}+\ldots+f_{0}^{\left(d_{m}\right)}$. Hence $\varphi^{\prime}\left(f_{0}^{\left(d_{i}\right)}\right)=f_{0}^{\left(d_{i}\right)}+R^{\prime}\left(x_{r_{i}+1}, x_{r+2}, \ldots, x_{n}\right)$ and by part (a) the coefficients $\lambda_{k l}$ in $\varphi\left(x_{k}\right)$ have the desired form. Now suppose some $c_{k} \neq 0$ for some $r_{i} \leq k \leq r_{i+1}-1$. Then, for a monomial $\mathbf{x}^{\alpha}$ in $R_{k}$, the term $\left(\lambda_{k j} x_{j}\right)^{d_{i}-1} \mathbf{x}^{\alpha}$ cannot be canceled from $\varphi\left(f_{0}^{\left(d_{i}\right)}+f_{0}^{\left(d_{i+1}\right)}+\ldots+f_{0}^{\left(d_{m}\right)}\right)$, a contradiction to $\varphi \in G_{f_{0}}^{\mathbf{w}}$. Hence we obtain

$$
\sum_{i=1}^{j} f_{0}^{\left(d_{i}\right)}=\varphi\left(\sum_{i=1}^{j} f_{0}^{\left(d_{i}\right)}\right)=\varphi\left(\sum_{i=1}^{j-1} f_{0}^{\left(d_{i}\right)}\right)+\varphi\left(f_{0}^{\left(d_{j}\right)}\right)=\sum_{i=1}^{j-1} f_{0}^{\left(d_{i}\right)}+\varphi\left(f_{0}^{\left(d_{j}\right)}\right),
$$

hence $f_{0}^{\left(d_{j}\right)}=\varphi\left(f_{0}^{\left(d_{j}\right)}\right)$ for $1 \leq j \leq r$.

Now we make use of the knowledge of the structure of $G_{f_{0}}^{\mathbf{w}}$ to show that the induced group-action of $G_{f_{0}}^{\mathbf{w}}$ on $T_{-}$is linear. 
Theorem 8. If $f_{0}$ is a Brieskorn-Pham polynomial then $E_{f_{0}} \subset G L_{k}(\mathbf{C})$, i.e., the induced action of $G_{f_{0}}^{\mathbf{w}}$ on $T_{-}$is linear.

Proof. By Theorem $5.7 G_{f_{0}}^{\mathbf{w}} \subset G L_{n}(\mathbf{C})$ is generated by diagonal and permutation matrices so we may assume that $f_{0}=\sum_{i=1}^{n} x_{i}^{d_{i}}$. For diagonal matrices $\sigma=\operatorname{diag}\left(\sigma_{1}, \sigma_{2}, \ldots, \sigma_{n}\right)$ it is clear that $\Theta(\sigma) \in E_{f_{0}}$ since

$$
\sigma^{-1} \bullet \mathbf{x}^{\alpha}=\left(\sigma_{1} x_{1}\right)^{\alpha_{1}} \cdot\left(\sigma_{2} x_{2}\right)^{\alpha_{2}} \cdot \ldots \cdot\left(\sigma_{n} x_{n}\right)^{\alpha_{n}}
$$

induces a diagonal matrix in $E_{f_{0}} \subset A u t\left(T_{-}\right)$. Now let $B_{-}$be the set of upper monomials of the Milnor algebra $M_{f_{0}}=\mathbf{C}\left[x_{1}, x_{2}, \ldots, x_{n}\right] /\left\langle x_{i}^{d_{i-1}}\right\rangle$. Since any permutation is a product of transpositions it suffices to consider the transposition $\pi=(i j)$. Note that any monomial in $B_{-}$can be written as $m=x_{i}^{\alpha_{i}} x_{j}^{\alpha_{j}} \cdot m^{\prime}$, where $m^{\prime}$ does not contain $x_{i}$ and $x_{j}$ and $\alpha_{i}<d_{i}-1, \alpha_{j}<d_{i}-1$. The application of the transposition $\pi \in G_{f_{0}}^{\mathbf{w}}$ to $m$ yields

$$
\pi \cdot\left(x_{i}^{\alpha_{i}} x_{j}^{\alpha_{j}} \cdot m^{\prime}\right)=x_{i}^{\alpha_{j}} x_{j}^{\alpha_{i}} \cdot m^{\prime} \in M_{-}
$$

since $\alpha_{i}, \alpha_{j}<d_{i}-1=d_{j}-1$ and induces a permutation matrix in $E_{f_{0}} \subset A u t\left(T_{-}\right)$. In both cases the map $\psi$ from Theorem 3.2 .3 is the identity.

Still the computation of moduli spaces for semiquasihomogeneous power series with principal part a Brieskorn-Pham polynomial may fail due to the intrinsic complexity of the invariant ring of $E_{f_{0}}$ and the quotient $T_{-} / E_{f_{0}}$. We illustrate the situation with 2 examples.

Example 6. Let $f_{0}=x^{4}+y^{8}$ be a Brieskorn-Pham polynomial of type $(8 ; 2,1)$. The semiuniversal family of negative weight is given by $F\left(x, y, t_{1}, t_{2}\right)=x^{4}+y^{8}+t_{1} x^{2} y^{6}+$ $t_{2} x^{2} y^{5}$. We have $\mu_{f_{0}}(0)=21,\left|G_{f_{0}}^{(2,1)}\right|=\left|E_{f_{0}}\right|=32$ and $T_{-}=\mathbf{C}^{2}$. The invariant ring equals $\mathbf{C}\left[t_{1}, t_{2}, t_{3}, t_{4}\right]^{G_{f_{0}}^{\mathrm{w}}}=\mathbf{C}\left[t_{1}^{4}, t_{1}^{2} t_{2}^{4}, t_{2}^{8}\right]$ and the embedding of the moduli space $\mathcal{M}=T_{-} / E_{f_{0}}$ in $\mathbf{C}^{3}$ is given by the variety $\mathcal{V}\left(y_{1} y_{3}-y_{2}^{2}\right)$.

Example 7. Let $f_{0}=x^{3}+y^{3}+z^{7}$ be a Brieskorn-Pham polynomial of type $(21 ; 7,7,3)$. We have $\mu_{f_{0}}(0)=24,\left|G_{f_{0}}^{(7,7,3)}\right|=\left|E_{f_{0}}\right|=126$ and $T_{-}=\mathbf{C}^{5}$. The invariant ring $\mathbf{C}\left[t_{1}, t_{2}, t_{3}, t_{4}, t_{5}\right]^{E_{f_{0}}}$ is generated by 162 homogeneous invariants $h_{1}, h_{2}, \ldots, h_{162}$ with degrees ranging from 3 to 42. Among the 162 generators delivered by Derksen's algorithm, 107 are invariant. Even the computation of the zeroset of $G_{f_{0}}^{(7,7,3)}$ takes ca. 2h25' (without applying the Reynolds operator) but we can write down the generators of $G_{f_{0}}^{\mathbf{w}}$ and compute the induced action by hand (or Singular 2.0). Then all matrices of $E_{f_{0}}$ are computed $(12.68 \mathrm{sec})$ and the Reynolds operator is applied to each non-invariant generator $(1589.30 \mathrm{sec})$. In order to compute the equations one has to eliminate the variables $t_{1}, t_{2}, t_{3}, t_{4}, t_{5}$ in the ideal $\left\langle y_{1}-h_{1}, y_{2}-h_{2}, \ldots, y_{162}-h_{162}\right\rangle$. 


\section{Conclusion}

We have described algorithms and their implementation in SINGULAR 2.0 for computing equations of moduli spaces of semiquasihomogeneous power series with principal part $f_{0}$ w.r.t. right equivalence. The algorithms are contained in the Singular 2.0 libraries qhmoduli.lib and rinvar.lib and some auxiliary algorithms are contained in zeroset.lib. The implementation is not restricted to specific classes of principal parts, but limitations arise from the intrinsic complexity of Gröbner bases and Arnold's Theorem.

If one considers the coarser relation contact equivalence instead of right equivalence, then a coarse moduli space still exists, but one has to fix more invariants and the construction is much more involved. In particular, the corresponding group need not be reductive! We refer to [9] for the existence proof. For a construction in the case of space curve singularities we refer to [6].

\section{References}

[1] Arnold, V.I. Normal forms of functions in neighborhoods of degenerate critical points. Usp. Mat. Nauk.29, No. 2, 1974.

[2] Arnold, V.I, Varchenko, A.N., Gusein-Zade, S.M. Singularities of differentiable mappings Vol. I,II. Birkhäuser, Basel 1985.

[3] Bayer, T. Computations of Moduli Spaces for semiquasihomogeneous Singularities and an Implementation in SINGULAR. Diploma Thesis, Fachbereich Mathematik, Universität Kaiserslautern (2000).

[4] Borel, A. Linear Algebraic Groups. 2nd Edition, Graduate Texts in Mathematics 126, Springer Verlag Berlin Heidelberg New York 1991.

[5] Derksen, H., Constructive Invariant Theory and the Linearization Problem. PhD thesis, University of Basel (2000).

[6] Frühbis-Krüger, A. Construction of Moduli Spaces for Space Curve Singularities of Multiplicity 3, Preprint, Universität Kaiserslautern (2000).

[7] Greuel, G.-M. elim.lib. A Singular 2.0 library for computing elimination, saturation and blowing up (1996).

[8] Greuel, G.-M. presolve.lib. A Singular 2.0 library for pre-solving of polynomial equations (2001).

[9] Greuel, G.-M., Hertling, K., Pfister, G. Moduli spaces of semiquasihomogeneous singularities with fixed principal part. J. Algebraic Geometry 6 (1997) 169 - 199.

[10] Greuel, G.-M., Pfister, G., and Schönemann, H. Singular 2.0. A Computer Algebra System for Polynomial Computations. Centre for Computer Algebra, University of Kaiserslautern (2001). http://www.singular.uni-kl.de.

[11] Heydtmann, A. E., finvar.lib. A Singular 2.0 library for computing invariant rings of finite groups (1997). 
[12] Harris, J., Morrison, I. Moduli of Curves Graduate Texts in Mathematics 187, Springer Verlag New York Berlin Heidelberg 1998.

[13] Kraft, H. Geometrische Methoden in der Invariantentheorie. 2. Auflage, Aspekte der Mathematik D1, Vieweg Verlag, Braunschweig 1985.

[14] Lamm, M. primitiv.lib. A Singular 2.0 library for computing a primitive element (1999).

[15] Orlik, P., Wagreich, P. Automorphisms of Singularities II. Math. Ann. 231 (1978) 229 -240 .

[16] G. Pfister, W. Decker and H. Schönemann, primdec.lib. A Singular 2.0 library for computing primary decompositions and radicals of ideals (2001).

[17] Saito, K. Einfach-elliptische Singularitäten. Invent. Math. 23 (1974) 289 - 325.

[18] Traverso, C. Hilbert Functions and the Buchberger Algorithm. J. Symb. Comp. Vol. 22 No. 4 (1996) 355 - 376.

[19] Vasconcelos, W. Computational Methods in Commutative Algebra and Algebraic Geometry. Algorithms and Computation in Mathematics Vol. 2, Springer Verlag Berlin Heidelberg New York 1997.

[20] Yoshinaga, E., Suzuki, M. Normal Forms of Non-Degnerate Quasihomogeneous Functions with Inner Modality $\leq 4$. Invent. Math. 55 (1979) 185 - 206. 